\title{
Vibration Analysis of Magneto-Electro-Thermo NanoBeam Resting on Nonlinear Elastic Foundation Using Sinc and Discrete Singular Convolution Differential Quadrature Method
}

\author{
Ola Ragb ${ }^{1}$, Mokhtar Mohamed ${ }^{2}$ \& M.S. Matbuly ${ }^{1}$ \\ ${ }^{1}$ Department of Engineering Mathematics and Physics, Faculty of Engineering, Zagazig University, Egypt \\ ${ }^{2}$ Basic science departement, Faculty of Engineering, Delta University for Science and Technology, Egypt \\ Correspondence: Mokhtar Mohamed, Faculty of Engineering, Delta University for Science and Technology, Egypt. \\ E-mail: mokhtar_husein@yahoo.com
}

Received: May 2, 2019

doi:10.5539/mas.v13n7p49
Accepted: June 8, 2019

Online Published: June 25, 2019

URL: https://doi.org/10.5539/mas.v13n7p49

\begin{abstract}
Magneto-Electro-Thermo nanobeam resting on a nonlinear elastic foundation is presented. This beam is subjected to the external electric voltage and magnetic potential, mechanical potential and temperature change. Also, we added the new material PTZ-5H-COFe2O4. The governing equations and boundary conditions are derived using Hamilton principle. These equations are discretized by using three differential quadrature methods and iterative quadrature technique to determine the natural frequencies and mode shapes. Numerical analysis is introduced to explain the influence of computational characteristics of the proposed schemes on convergence, accuracy and efficiency of the obtained results. The obtained results agreed with the previous analytical and numerical ones. A detailed parametric study is conducted to investigate the influences of different boundary conditions, various composite materials, nonlinear elastic foundation, nonlocal parameter, the length-to-thickness ratio, external electric and magnetic potentials, axial forces, temperature and their effects on the vibration characteristics of Magneto-Electro-Thermo-Elastic nanobeam.
\end{abstract}

Keywords: composite material, differential quadrature, discrete singular convolution, nonlinear elastic foundation, nonlocal elasticity theory, piezo magnetic, sinc, timoshenko theory, vibration

\section{Introduction}

Magneto-Electro-Thermo-Elastic (METE) composite materials which have piezoelectric and piezomagnetic phases can convert electric, thermal, elastic and magnetic energies to another form (Jandaghian, A., Jafari, A., \& Rahmani, O., 2013; Nan, C. W., 1994). Many technological fields use METE materials such as vibration control, actuator applications, sensor, medical instruments, health monitoring and energy harvesting (Zhai et al., 2008; Nan et al., 2008). Wu et al., (2007) investigated three-dimensional (3D) static behavior of doubly curved functionally graded (FG) magneto-electro-elastic shells under mechanical load, electric displacement and magnetic flux. Huang et al., (2010) studied the analytical and semi-analytical solutions for anisotropic functionally graded magnetoelectro-elastic beam subjected to an arbitrary load, which can be expanded in terms of sinusoidal series. (Chang, 2013) presented the free vibration, deterministic vibration and random vibration characteristics of transversely isotropic magneto-electro-elastic rectangular plates in contact with the fluid. Ansari et al., (2015) developed a nonlocal geometrically nonlinear beam model for magneto-electro-thermo-elastic nanobeams subjected to external electric voltage, external magnetic potential and uniform temperature rise. Ke et al., (2014b) studied the free vibration of embedded magneto-electro- elastic cylindrical nanoshells based on Love's shell theory. Research communities concerned with METE nanomaterials and their nanostructures (BiFeO3, BiTiO3-CoFe2O4, NiFe2O4-PZT, nanowires, nanobeams) (Prashanthi et al., 2012; Martin et al., 2008). Also, we added the new material PTZ-5H-COFe2O4.

The nonlocal theory of elasticity is widely used for the study of nanoscale problems. Vibrations of nanobeams have been the theme of some experiments and molecular dynamics simulations. As experiments at the nanoscale are extremely difficult computations remain expensive for large size atomic systems, continuum models continue to play an essential role in the study of nanostructures. However, there are strong evidences that the small length scale effect i.e., nonlocal effect has a significant influence on the mechanical behavior of nanostructures. Therefore, 
classical structural theories have to be modified to use for the small length scale effect (Akgöz, B., \& Civalek, O., 2011; Li, 2014; Shen et al., 2012; Li, X. F., \& Wang, B. L., 2009; Huang et al., 2013; Shen, J. P., \& Li, C., 2017; Mercan, K., \& Civalek, O., 2016).

Many papers, dealing with static and dynamic behavior of METE nanobeams, have been published recently. The exact and semi analytical method are presented for solving the linear free and forced vibration, buckling of nanobeams (Arefi, M., \& Zenkour, A.M., 2016; Şimşek, M., \& Yurtcu, H., 2013; Jian et al., 2010). In another work, the numerical method such as finite element (Norouzzadeh, A., \& Ansari, R., 2017), meshless (Roque et al., 2013), higher order B-spline finite strip (Foroughi, H., \& Azhari, M., 2014), Rayleigh-Ritz (Fakher, M., \& Shahrokh, H. H., 2017) are examined for solving this problem. But all these methods need a large number of grid points as well as a large computer capacity to attain a considerable accuracy.

A polynomial based differential quadrature method (PDQM) leads to accurate solutions with fewer grid points (Shojaei, M. F., \& Ansari, R., 2017; Tornabene et al., 2014; Tornabene et al., 2016) comparing to other numerical methods. Sinc differential quadrature method (SDQM) (Ke et al., 2012), and Discrete singular convolution differential quadrature method (DSCDQM) (Korkmaz, A., \& İdris, D., 2011) are more reliable versions than polynomial based DQM. These methods depend on choice of shape function such that Cardinal sine function, Delta Lagrange Kernel (DLK) and Regularized Shannon kernel (RSK) are shape functions which gives more convergence and stability for the results (Seçkin, A., \& Sarıgül, A. S., 2009; Civalek, 2008; Civalek, 2009; Civalek, O. \& Kiracioglu, O., 2010; Civalek, 2017) .

According to the knowledge of the authors, SDQM and DSCDQM are not examined for vibration analysis of Magneto-Electro-Thermo nanobeam resting on the nonlinear elastic foundation. Also, the present work extends the applications of DQM to analyze this problem. Based on these versions, numerical schemes are designed for vibration of METE nanobeam. MATLAB program is designed to solve this problem. The natural frequencies are obtained and compared with previous analytical and numerical ones. For each scheme the convergence and efficiency are verified. Also, a parametric study is introduced to investigate the influence of linear and nonlinear elastic foundation, temperature change, external electric voltage, different boundary conditions and materials, nonlocal parameter, axial forces, external magnetic potential and length-to-thickness ratio on the values of natural frequencies and mode shapes.

\section{Formulation of the Problem}

Consider a Magneto-Electro-Thermo-Elastic nanobeam with $(0 \leq x \leq L, \quad 0 \leq z \leq h)$ where $\mathrm{L}$ and $\mathrm{h}$ are length and thickness of the beam. This beam is polarized in $\mathrm{z}$ direction and subjected to an applied voltage $\phi(x, z, t)$, a magnetic potential $\psi(x, z, t)$ a uniform temperature change $\Delta T$, mechanical potential $P_{0}$ frand linear and nonlinear elastic foundation $K_{1}, K_{2}, K_{3}$ respectively as shown in Figure. (1).

The equations of motion can be written by using Hamilton principle as (Shojaei, M. F., \& Ansari, R., 2017):

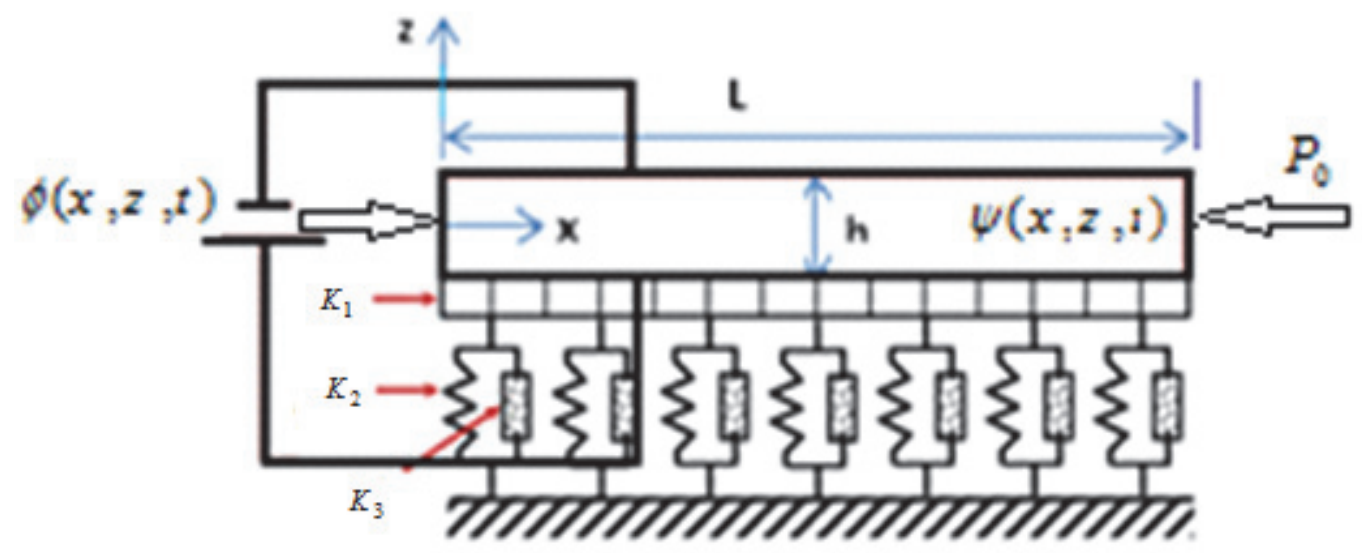

Figure 1. Magneto-Electro-Thermo Nanobeam resting on on nonlinear elastic foundation 


$$
\begin{aligned}
& \mathrm{k}_{\mathrm{s}} \mathrm{A}_{44}\left[\frac{\partial^{2} w}{\partial \mathrm{x}^{2}}+\frac{\partial \theta_{x}}{\partial \mathrm{x}}\right]+\left(\mathrm{N}_{P}+\mathrm{N}_{\mathrm{E}}+\mathrm{N}_{\mathrm{T}}+\mathrm{N}_{M}\right)\left(\frac{\partial^{2} w}{\partial \mathrm{x}^{2}}-\left(e_{0} a\right)^{2} \frac{\partial^{4} w}{\partial \mathrm{x}^{4}}\right)-K_{1}\left[w-\left(e_{0} a\right)^{2} \frac{\partial^{2} w}{\partial \mathrm{x}^{2}}\right] \\
& +K_{2}\left[\frac{\partial^{2} w}{\partial \mathrm{x}^{2}}-\left(e_{0} a\right)^{2} \frac{\partial^{4} w}{\partial \mathrm{x}^{4}}\right]+K_{3} w^{3}-\mathrm{k}_{\mathrm{s}}\left[E_{15} \frac{\partial^{2} \phi}{\partial \mathrm{x}^{2}}+Q_{15} \frac{\partial^{2} \psi}{\partial \mathrm{x}^{2}}\right]=\mathrm{I}_{0} \frac{\partial^{2}}{\partial \mathrm{t}^{2}}\left[w-\left(\mathrm{e}_{0} \mathrm{a}\right)^{2} \frac{\partial^{2} w}{\partial \mathrm{x}^{2}}\right] \\
& \mathrm{D}_{11} \frac{\partial^{2} \theta_{x}}{\partial \mathrm{x}^{2}}-\mathrm{k}_{\mathrm{s}} \mathrm{A}_{44}\left(\frac{\partial w}{\partial \mathrm{x}}+\theta_{x}\right)+\left(E_{31}+\mathrm{k}_{\mathrm{s}} \mathrm{E}_{15}\right) \frac{\partial \phi_{E}}{\partial \mathrm{x}}+\left(Q_{31}+\mathrm{k}_{\mathrm{s}} Q_{15}\right) \frac{\partial \psi_{H}}{\partial \mathrm{x}}=\mathrm{I}_{2} \frac{\partial^{2}}{\partial \mathrm{t}^{2}}\left[\theta_{x}-\left(\mathrm{e}_{0} \mathrm{a}\right)^{2} \frac{\partial^{2} \theta_{x}}{\partial \mathrm{x}^{2}}\right], \\
& E_{31} \frac{\partial \theta_{x}}{\partial \mathrm{x}}+\mathrm{E}_{15}\left[\frac{\partial^{2} w}{\partial \mathrm{x}^{2}}+\frac{\partial \theta_{x}}{\partial \mathrm{x}}\right]+\mathrm{X}_{11} \frac{\partial^{2} \phi_{E}}{\partial \mathrm{x}^{2}}+Y 11 \frac{\partial^{2} \psi_{H}}{\partial \mathrm{x}^{2}}-\mathrm{X}_{33} \phi_{E}-Y_{33} \psi_{H}=0, \\
& Q_{31} \frac{\partial \theta_{x}}{\partial \mathrm{x}}+Q_{15}\left[\frac{\partial^{2} w}{\partial \mathrm{x}^{2}}+\frac{\partial \theta_{x}}{\partial \mathrm{x}}\right]+Y_{11} \frac{\partial^{2} \phi_{E}}{\partial \mathrm{x}^{2}}+T_{11} \frac{\partial^{2} \psi_{H}}{\partial \mathrm{x}^{2}}-Y_{33} \phi_{E}-T_{33} \psi_{H}=0,
\end{aligned}
$$

By putting the electric potential and magnetic potential are zero at the ends of the nanobeam (Ke et al., 2012). The boundary conditions can be written as

(1) For Clamped end (C):

$$
\theta(0, t)=w(0, t)=\phi(0, t)=\psi(0, t)=0, \quad \theta(L, t)=w(L, t)=\phi(L, t)=\psi(L, t)=0
$$

(2) For Hinged end $(\mathrm{H})$ :

$$
\begin{aligned}
& w(0, t)=\phi(0, t)=\psi(0, t)=0, \quad w(L, t)=\phi(L, t)=\psi(L, t)=0, \\
& \overline{\mathrm{D}}_{11} \frac{\partial \theta(0, t)}{\partial x}+\bar{E}_{31}(\phi(0, t)+\psi(0, t))-\omega^{2}\left(e_{0} a\right)^{2}\left[\overline{\mathrm{I}}_{2} \frac{\partial \theta(0, t)}{\partial x}+\overline{\mathrm{I}}_{0} w(0, t)\right]- \\
& \left(\overline{\mathrm{N}}_{\mathrm{E}}+\overline{\mathrm{N}}_{\mathrm{T}}+\bar{N}_{P}+\bar{N}_{M}\right)\left(e_{0} a\right)^{2} \frac{\partial^{2} w(0, t)}{\partial x^{2}}+\left(e_{0} a\right)^{2}\left(k_{1} w(0, t)-k_{2} \frac{\partial^{2} w(0, t)}{\partial x^{2}}+k_{3^{w}}{ }^{3}(0, t)\right)=0, \\
& \overline{\mathrm{D}}_{11} \frac{\partial \theta(L, t)}{\partial x}+\bar{E}_{31}(\phi(L, t)+\psi(L, t))-\omega^{2}\left(e_{0} a\right)^{2}\left[\overline{\mathrm{I}}_{2} \frac{\partial \theta(L, t)}{\partial x}+\overline{\mathrm{I}}_{0} w(L, t)\right] \\
& -\left(\overline{\mathrm{N}}_{\mathrm{E}}+\overline{\mathrm{N}}_{\mathrm{T}}+\bar{N}_{P}+\bar{N}_{M}\right)\left(e_{0} a\right)^{2} \frac{\partial^{2} w(L, t)}{\partial x^{2}}+\left(e_{0} a\right)^{2}\left(k_{1} w(L, t)-k_{2} \frac{\partial^{2} w(L, t)}{\partial x^{2}}+k_{3^{w}}{ }^{3}(L, t)\right)=0,
\end{aligned}
$$

Where $\mathrm{w}, \theta_{\mathrm{X}}, \mathrm{f}$ and $\psi$ are displacement in mid-plane, cross section rotation, electric potential and magnetic potential respectively; $\left(e_{0} a\right)$ is the scale coefficient which incorporates the small-scale effect. $k_{S}$ is the shear correction factor which takes values 5/6 for the macro scale beams (Shojaei, M. F., \& Ansari, R., 2017; Tornabene et al., 2014; Tornabene et al., 2016).

$$
\mathrm{N}_{\mathrm{T}}=-\bar{\lambda}_{1} \mathrm{~h} \Delta \mathrm{T}, \mathrm{N}_{\mathrm{E}}=2 \overline{\mathrm{e}}_{31} \mathrm{~V}_{0}, \mathrm{~N}_{P}=P_{0}, \mathrm{~N}_{M}=2 \bar{q}_{31} A_{0}
$$

Where $N_{E}, N_{M}, N_{T}$ and $N_{P}$ are normal forces induced by the external electric potential $\mathrm{v}_{0}$, external magnetic potential $A_{0}$, temperature change $\Delta T$ and mechanical potential $P_{0} \cdot \bar{\lambda}_{1}, \overline{\mathrm{e}}_{31}$ and $\overline{\mathrm{q}}_{31}$ are thermal module, 
piezoelectric constant and piezomagnetic constant, respectively; $K_{1}, K_{2}, K_{3}$ are shear and spring coefficients of linear and nonlinear elastic foundation (Jandaghian, A. A., \& Rahmani, O., 2016).

Also,

$$
\begin{aligned}
& \left(\mathrm{A}_{11}, \mathrm{~A}_{44}\right)=\left(\overline{\mathrm{C}}_{11}, \overline{\mathrm{C}}_{44}\right) \mathrm{h}, \mathrm{D}_{11}=\overline{\mathrm{C}}_{11} \mathrm{~h}^{3} / 12,\left(\mathrm{E}_{15}, \mathrm{Q}_{15}\right)=2 \frac{\left(\overline{\mathrm{e}}_{15}, \overline{\mathrm{q}}_{15}\right)}{\beta} \sin \left(\frac{\beta \mathrm{h}}{2}\right),\left(\mathrm{I}_{0}, \mathrm{I}_{2}\right)=\rho\left(\mathrm{h}, \mathrm{h}^{3} / 12\right), \\
& \left(E_{31}, \mathrm{Q}_{31}\right)=\left(\overline{\mathrm{e}}_{31}, \overline{\mathrm{q}}_{31}\right)\left[-\mathrm{h} \cos \left(\frac{\beta \mathrm{h}}{2}\right)+\frac{2}{\beta} \sin \left(\frac{\beta \mathrm{h}}{2}\right)\right],\left(\mathrm{X}_{11}, \mathrm{Y}_{11}, \mathrm{~T}_{11}\right)=\frac{\left(\bar{S}_{11}, \bar{d}_{11}, \bar{\mu}_{11}\right)}{2}\left[\mathrm{~h}+\frac{\sin (\beta \mathrm{h})}{\beta}\right], \\
& \left(\mathrm{X}_{33}, \mathrm{Y}_{33}, \mathrm{~T}_{33}\right)=\frac{\left(\bar{S}_{33}, \bar{d}_{33}, \bar{\mu}_{33}\right) \beta^{2}}{2}\left[\mathrm{~h}-\frac{\sin (\beta \mathrm{h})}{\beta}\right], \beta=\pi / \mathrm{h}
\end{aligned}
$$

And

$$
\begin{aligned}
& \left(\bar{C}_{11}, \bar{C}_{44}\right)=\left(C_{11}-\frac{C_{13}^{2}}{C_{33}}, C_{44}\right),\left(\bar{e}_{31}, \bar{e}_{15}\right)=\left(e_{31}-\frac{C_{13} e_{33}}{C_{33}}, \mathrm{e}_{15}\right),\left(\bar{q}_{31}, \bar{q}_{15}\right)=\left(q_{31}-\frac{C_{13} q_{33}}{C_{33}}, \mathrm{q}_{15}\right), \\
& \left(\bar{S}_{33}, \bar{S}_{11}\right)=\left(S_{33}-\frac{e_{33}^{2}}{C_{33}}, \mathrm{~S}_{11}\right),\left(\bar{d}_{33}, \overline{\mathrm{d}}_{11}\right)=\left(d_{33}+\frac{q_{33} e_{33}}{C_{33}}, \mathrm{~d}_{11}\right),\left(\bar{\mu}_{33}, \bar{\mu}_{11}\right)=\left(\mu_{33}+\frac{q_{33}^{2}}{C_{33}}, \mu_{11}\right), \\
& \left(\bar{\lambda}_{1}, \overline{\mathrm{p}}_{3}, \bar{\beta}_{3}\right)=\left(\lambda_{1}-\frac{C_{13} \lambda_{3}}{C_{33}}, \mathrm{p}_{3}+\frac{\lambda_{3} e_{33}}{C_{33}}, \bar{\beta}_{3}+\frac{\beta_{3} q_{33}}{C_{33}}\right)
\end{aligned}
$$

Where $\bar{C}_{i j}, \overline{\mathrm{e}}_{i j}, \overline{\mathrm{s}}_{i j}, \overline{\mathrm{q}}_{i j}, \overline{\mathrm{d}}_{i j}, \bar{\mu}_{i j}, \bar{\lambda}_{i}, \overline{\mathrm{p}}_{i}$ and $\bar{\beta}_{i}$ are elastic, piezoelectric, dielectric, piezo-magnetic,

magneto-electric, magnetic, thermal moduli, pyro-electric and pyro-magnetic constants.

The field quantities are normalized such as :

$$
\begin{aligned}
& \zeta=\frac{\mathrm{x}}{\mathrm{L}}, \mathrm{w}=\frac{\mathrm{W}}{L}, \eta=\frac{\mathrm{L}}{\mathrm{h}}, \mu=\frac{\mathrm{e}_{0}^{\mathrm{a}}}{\mathrm{L}}, \theta_{x}=\Theta, \varphi=\frac{\phi_{E}}{\phi_{0}}, \phi_{0}=\sqrt{\frac{\mathrm{A}_{11}}{X_{33}}}, \psi_{0}=\sqrt{\frac{\mathrm{A}_{11}}{T_{33}}}, \overline{\mathrm{A}}_{44}=\frac{\mathrm{A}_{44}}{\mathrm{~A}_{11}}, \\
& \overline{\mathrm{D}}_{11}=\frac{\mathrm{D}_{11}}{\mathrm{~A}_{11} \mathrm{~h}^{2}}, \overline{\mathrm{I}}_{0}=\frac{\mathrm{I}_{0}}{\mathrm{I}_{0}}, \overline{\mathrm{I}}_{2}=\frac{\mathrm{I}_{2}}{\mathrm{I}_{0} \mathrm{~h}^{2}}, \overline{\mathrm{x}}_{11}=\frac{\mathrm{X}_{11} \phi_{0}^{2}}{\mathrm{~A}_{11} \mathrm{~h}^{2}}, \overline{\mathrm{x}}_{33}=\frac{\mathrm{X}_{33} \phi_{0}^{2}}{\mathrm{~A}_{11}}, \overline{\mathrm{E}}_{15}=\frac{\mathrm{E}_{15} \phi_{0}}{\mathrm{~A}_{11} \mathrm{~h}}, \bar{E}_{31}=\frac{E_{31} \phi_{0}}{\mathrm{~A}_{11} \mathrm{~h}} \\
& \bar{Q}_{15}=\frac{Q_{15} \psi_{0}}{\mathrm{~A}_{11} \mathrm{~h}}, \bar{Q}_{31}=\frac{Q_{31} \psi_{0}}{\mathrm{~A}_{11} \mathrm{~h}}, \overline{\mathrm{N}}_{\mathrm{T}}=-\frac{\lambda 1 \mathrm{~h} \Delta \mathrm{T}}{\mathrm{A}_{11}}, \overline{\mathrm{N}}_{\mathrm{E}}=\frac{2 \mathrm{e}_{31} \mathrm{~V}_{0}}{\mathrm{~A}_{11}}, \overline{\mathrm{N}}_{p}=\frac{p_{0}}{\mathrm{~A}_{11}}, \overline{\mathrm{N}}_{M}=\frac{2 q_{31} A_{0}}{\mathrm{~A}_{11}}, \\
& \tau=\frac{\mathrm{t}}{\mathrm{L}} \sqrt{\frac{\mathrm{I}_{0}}{\mathrm{~A}_{11}}}, \mathrm{k}_{1}=\frac{K_{1} L^{4}}{\pi^{2} \mathrm{~A}_{11} \mathrm{~h}^{2}}, \mathrm{k}_{2}=\frac{K_{2} L^{2}}{\pi^{2} \mathrm{~A}_{11} \mathrm{~h}^{2}}, \mathrm{k}_{3}=\frac{K_{3} L^{2}}{\pi^{2} \mathrm{~A}_{11} \mathrm{~h}^{2}},
\end{aligned}
$$

Furthermore, for harmonic behavior, one can assume that:

$w(x, t)=W e^{i \omega t}, \quad \theta(x, t)=\Theta e^{i \omega t}, \quad \psi(x, t)=\Psi e^{i \omega t}, \quad \phi(x, t)=\Phi e^{i \omega t}$

Where $\omega$ is the natural frequency of the beam and $i=\sqrt{-1}$.

The amplitudes for $w, \theta, \psi$ and $\phi$ are $W, \Theta, \Psi$ and $\Phi$ respectively.

Substituting from equations (12-13) into (1-4), the problem can be reduced to a static one as: 


$$
\begin{aligned}
& \mathrm{k}_{\mathrm{s}} \overline{\mathrm{A}}_{44}\left[\frac{\partial^{2} W}{\partial \zeta^{2}}+\eta \frac{\partial \Theta}{\partial \zeta}\right]-\mathrm{k}_{\mathrm{s}}\left(\overline{\mathrm{E}}_{15} \frac{\partial^{2} \Phi}{\partial \zeta^{2}}+\bar{Q}_{15} \frac{\partial^{2} \Psi}{\partial \zeta^{2}}\right)+\left(\overline{\mathrm{N}}_{\mathrm{E}}+\overline{\mathrm{N}}_{\mathrm{T}}+\overline{\mathrm{N}}_{p}+\overline{\mathrm{N}}_{m}\right)\left(\frac{\partial^{2} W}{\partial \zeta^{2}}-\mu^{2} \frac{\partial^{4} W}{\partial \zeta^{4}}\right) \\
& -k_{1}\left(W-\mu^{2} \frac{\partial^{2} W}{\partial \zeta^{2}}\right)+k_{2}\left(\frac{\partial^{2} W}{\partial \zeta^{2}}-\mu^{2} \frac{\partial^{4} W}{\partial \zeta^{4}}\right)+k_{3} W^{3}=-\omega^{2} \overline{\mathrm{I}}_{0}\left[W-\mu^{2} \frac{\partial^{2} W}{\partial \zeta^{2}}\right], \\
& \overline{\mathrm{D}}_{11} \frac{\partial^{2} \Theta}{\partial \zeta^{2}}-\mathrm{k}_{\mathrm{s}} \overline{\mathrm{A}}_{44} \eta\left(\frac{\partial W}{\partial \zeta}+\eta \Theta\right)+\left(\bar{E}_{31}+\mathrm{k}_{\mathrm{s}} \overline{\mathrm{E}}_{15}\right) \eta \frac{\partial \Phi}{\partial \zeta}+\left(\bar{Q}_{31}+\mathrm{k}_{\mathrm{s}} \bar{Q}_{15}\right) \eta \frac{\partial \Psi}{\partial \zeta}=-\omega^{2} \overline{\mathrm{I}}_{2}\left[\Theta-\mu^{2} \frac{\partial^{2} \Theta}{\partial \zeta^{2}}\right] \\
& \bar{E}_{31} \eta \frac{\partial \Theta}{\partial \zeta}+\overline{\mathrm{E}}_{15}\left[\frac{\partial^{2} W}{\partial \zeta^{2}}+\eta \frac{\partial \Theta}{\partial \zeta}\right]+\overline{\mathrm{X}}_{11} \frac{\partial^{2} \Phi}{\partial \zeta^{2}}-\overline{\mathrm{X}}_{33} \eta^{2} \Phi+\bar{Y}_{11} \frac{\partial^{2} \Psi}{\partial \zeta^{2}}-\bar{Y}_{33} \eta^{2} \Psi=0 \\
& \bar{Q}_{31} \eta \frac{\partial \Theta}{\partial \zeta}+\bar{Q}_{15}\left[\frac{\partial^{2} W}{\partial \zeta^{2}}+\eta \frac{\partial \Theta}{\partial \zeta}\right]+\bar{Y}_{11} \frac{\partial^{2} \Phi}{\partial \zeta^{2}}-\bar{Y}_{33} \eta^{2} \Phi+\bar{T}_{11} \frac{\partial^{2} \Psi}{\partial \zeta^{2}}-\bar{T}_{33} \eta^{2} \Psi=0
\end{aligned}
$$

Substituting from equations (12-13) into (5-8), the boundary conditions can be written as:

(1) For Clamped end (C): $\quad \Theta=W=\Phi=\Psi=0, \quad \zeta=0,1$

(2) For Hinged end (H): $\quad W=\Phi=\Psi=0, \quad \zeta=0,1$

$\overline{\mathrm{D}}_{11} \frac{\partial \Theta}{\partial \zeta}+{\overline{E_{31}}}_{31} \eta(\Phi+\Psi)-\omega^{2} \mu^{2}\left[\overline{\mathrm{I}}_{2} \frac{\partial \Theta}{\partial \zeta}+\overline{\mathrm{I}}_{0} \eta \mathrm{W}\right]$

$-\eta\left(\overline{\mathrm{N}}_{\mathrm{E}}+\overline{\mathrm{N}}_{\mathrm{T}}+\bar{N}_{P}+\bar{N}_{M}\right) \mu^{2} \frac{\partial^{2} W}{\partial \zeta^{2}}+\mu^{2}\left(k_{1} W-k_{2} \eta \frac{\partial^{2} W}{\partial \zeta^{2}}+k_{3}{ }^{3}\right)=0$,

\section{Method of Solution}

Three differential quadrature techniques and iterative quadrature technique are employed to reduce the governing equation into an Eigen value problem as follows:

\section{- Polynomial based differential quadrature method (PDQM)}

In this technique, Lagrange interpolation polynomial is employed as a shape function such that the unknown $\mathrm{v}$ and its derivatives can be approximated as a weighted linear sum of nodal values, $\mathrm{v}_{i},(\mathrm{i}=1: \mathrm{N})$, as follows (Chang, 2000):

$v\left(x_{i}\right)=\sum_{j=1}^{N} \frac{\prod_{k=1}^{N}\left(x_{i}-x_{k}\right)}{\left(x_{i}-x_{j}\right) \prod_{j=1, j \neq k}^{N}\left(x_{j}-x_{k}\right)} v\left(x_{j}\right), \quad(i=1: N)$,
$\left.\frac{\partial v}{\partial x}\right|_{x=x_{i}}=\sum_{j=1}^{N} C_{i j}^{(1)} v_{j}\left(x_{j}\right),\left.\quad \frac{\partial^{2} v}{\partial x^{2}}\right|_{x=x_{i}}=\sum_{j=1}^{N} C_{i j}^{(2)} v_{j}\left(x_{j}\right), \quad(i=1: N)$

Similarly, one can approximate $\frac{\partial^{3} v}{\partial x^{3}}, \frac{\partial^{4} v}{\partial x^{4}}$ and calculate $C_{i j}^{(3)}, C_{i j}^{(4)}$ 
Where $v$ terms to $W, \Theta, \Phi$ and $\Psi \cdot N$ is the number of grid points. The weighting coefficients $C_{i j}^{(1)}$ can be determined by differentiating (21) as (Chang, 2000):

$C_{i j}^{(1)}= \begin{cases}\frac{1}{\left(x_{i}-x_{j}\right)} \prod_{k=1, k \neq i, j}^{N} \frac{\left(x_{i}-x_{k}\right)}{\left(x_{j}-x_{k}\right)} & i \neq j \\ -\sum_{j=1, j \neq i}^{N} C_{i j}^{(1)} & i=j\end{cases}$

Also, by using matrix multiplication can be calculated $C_{i j}^{(2)}, C_{i j}^{(3)}$ and $C_{i j}^{(4)}$ as:

$\left[C_{i j}^{(n)}\right]=\left[C_{i j}^{(1)}\right]\left[C_{i j}^{(n-1)}\right],(n=2,3,4)$

\section{- $\quad$ Sinc Differential Quadrature Method (SDQM)}

A Cardinal sine function is used as a shape function such that the unknown $v$ and its derivatives can be approximated as a weighted linear sum of nodal values, $v_{i},(i=-N: N)$, as follows:

$S_{j}\left(x_{i}, h_{x}\right)=\frac{\sin \left[\pi\left(x_{i}-x_{j}\right) / h_{x}\right]}{\pi\left(x_{i}-x_{j}\right) / h_{x}}$, where $\left(\mathrm{h}_{\mathrm{x}}>0\right)$ is the step size.

This function is applied as a shape function such that the unknown $v$ and its derivatives are approximated as a weighted linear sum of nodal values, $v_{i},(i=-N: N)$, as follows (Korkmaz, A., \& İdris, D., 2011):

$v\left(x_{i}\right)=\sum_{j=-N}^{N} \frac{\sin \left[\pi\left(x_{i}-x_{j}\right) / h_{x}\right]}{\pi\left(x_{i}-x_{j}\right) / h_{x}} v\left(x_{j}\right), \quad(i=-N: N), h_{x}>0$

$\left.\frac{\partial v}{\partial x}\right|_{x=x_{i}}=\sum_{j=-N}^{N} C_{i j}^{(1)} v\left(x_{j}\right),\left.\quad \frac{\partial^{2} v}{\partial x^{2}}\right|_{x=x_{i}}=\sum_{j=-N}^{N} C_{i j}^{(2)} v\left(x_{j}\right), \quad(i=-N: N)$,

Similarly, one can approximate $\frac{\partial^{3} v}{\partial x^{3}}, \frac{\partial^{4} v}{\partial x^{4}}$ and calculate $C_{i j}^{(3)}, C_{i j}^{(4)}$

Where $v$ terms to $W, \Theta, \Phi$ and $\Psi . N$ is the number of grid points. $\mathrm{h}_{\mathrm{x}}$ is grid size. The weighting coefficients $C_{i j}^{(1)}, C_{i j}^{(2)}, C_{i j}^{(3)}$ and $C_{i j}^{(4)}$ can be determined by differentiating (25) and (26) as:

$$
\begin{aligned}
& C_{i j}^{(1)}=\left\{\begin{array}{ll}
\frac{(-1)^{i-j}}{h_{x}(i-j)}, & i \neq j \\
0 & i=j
\end{array}, \quad \mathrm{C}_{\mathrm{ij}}^{(2)}= \begin{cases}\frac{2(-1)^{\mathrm{i}-\mathrm{j}+1}}{h_{x}{ }^{2}(\mathrm{i}-\mathrm{j})^{2}}, & i \neq j \\
-\frac{\pi^{2}}{3 h_{x}{ }^{2}} & i=j\end{cases} \right. \\
& \mathrm{C}_{\mathrm{ij}}^{(3)}=\left\{\begin{array}{ll}
\frac{(-1)^{\mathrm{i}-\mathrm{j}}}{h_{x}{ }^{3}(\mathrm{i}-\mathrm{j})^{3}}\left(6-\pi^{2}(\mathrm{i}-\mathrm{j})^{2}\right), & i \neq j \\
0 & i=j
\end{array}, \mathrm{C}_{\mathrm{ij}}^{(4)}= \begin{cases}\frac{4(-1)^{\mathrm{i}-\mathrm{j}+1}}{h_{x}{ }^{4}(\mathrm{i}-\mathrm{j})^{4}}\left(6-\pi^{2}(\mathrm{i}-\mathrm{j})^{2}\right), & i \neq j \\
\frac{\pi^{4}}{5 h_{x}{ }^{4}} & i=j\end{cases} \right.
\end{aligned}
$$




\section{- Discrete Singular Convolution Differential Quadrature Method (DSCDQM)}

A singular convolution can be defined as (Seçkin, A., \& Sarıgül, A. S., 2009; Civalek, 2008; Civalek, 2009; Civalek, O. \& Kiracioglu, O., 2010; Civalek, 2017)

$$
F_{(t)}=\left(T^{*} \eta\right)(t)=\int_{-\infty}^{\infty} T(t-x) \eta(x) d x
$$

Where $T(t-x)$ is a singular kernel.

The DSC algorithm can be applied using many types of kernels. These kernels are applied as shape functions such that the unknown $v$ and its derivatives are approximated as a weighted linear sum of $v_{i,}(i=-N: N)$, over a narrow bandwidth $\left(x-x_{M}, x+x_{M}\right)$.

Two kernels of DSC will be employed as follows:

(a) Delta Lagrange Kernel (DLK) can be used as a shape function such that the unknown $\mathrm{v}$ and its derivatives can be approximated as a weighted linear sum of nodal values, $\mathrm{v}_{\mathrm{i}},(\mathrm{i}=-\mathrm{N}: \mathrm{N})$, as follows :

$$
\begin{gathered}
v\left(x_{i}\right)=\sum_{j=-M}^{M} \frac{\prod_{k=-M}^{M}\left(x_{i}-x_{k}\right)}{\left(x_{i}-x_{j}\right) \prod_{j=-M, j \neq k}^{M}\left(x_{j}-x_{k}\right)} v\left(x_{j}\right), \quad(i=-N: N), M \geq 1 \\
\left.\frac{\partial v}{\partial x}\right|_{x=x_{i}}=\sum_{j=-M}^{M} C_{i j}^{(1)} v\left(x_{j}\right),\left.\quad \frac{\partial^{2} v}{\partial x^{2}}\right|_{x=x_{i}}=\sum_{j=-M}^{M} C_{i j}^{(2)} v\left(x_{j}\right), \quad(i=-N: N),
\end{gathered}
$$

Similarly, one can approximate $\frac{\partial^{3} v}{\partial x^{3}}, \frac{\partial^{4} v}{\partial x^{4}}$ and calculate $C_{i j}^{(3)}, C_{i j}^{(4)}$

Where $2 \mathrm{M}+1$ is the effective computational bandwidth.

$\mathrm{C}_{\mathrm{ij}}^{(1)}, \mathrm{C}_{\mathrm{ij}}^{(2)}, \mathrm{C}_{\mathrm{ij}}^{(3)}$ and $\mathrm{C}_{\mathrm{ij}}^{(4)}$ are defined as :

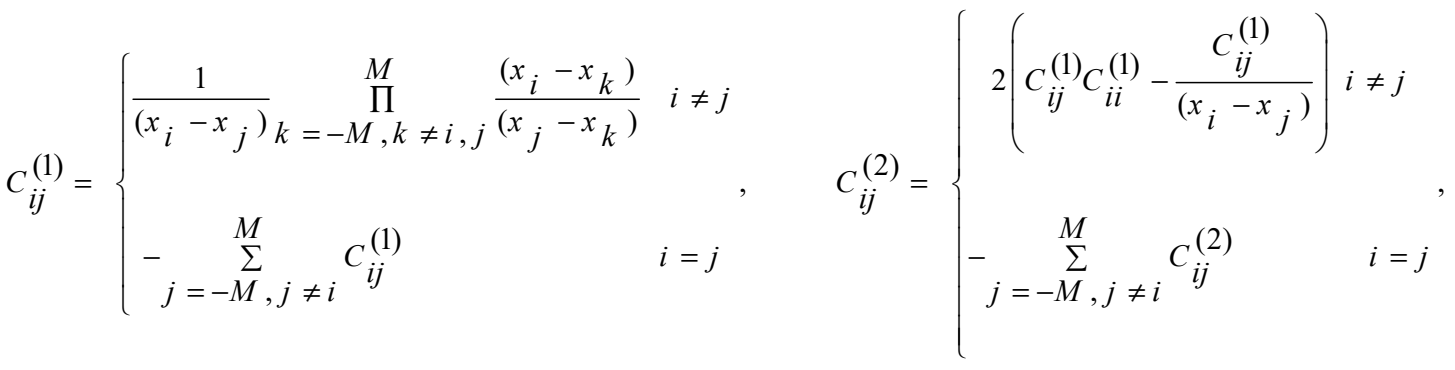

$$
\begin{aligned}
& C_{i j}^{(3)}=\left\{\begin{array}{ll}
3\left(C_{i j}^{(1)} C_{i i}^{(2)}-\frac{C_{i j}^{(2)}}{\left(x_{i}-x_{j}\right)}\right) & i \neq j \\
-\sum_{j=-M, j \neq i}^{M} C_{i j}^{(3)} & i=j
\end{array} \quad C_{i j}^{(4)}=\left\{\begin{array}{l}
4\left(C_{i j}^{(1)} C_{i i}^{(3)}-\frac{C_{i j}^{(3)}}{\left(x_{i}-x_{j}\right)}\right) i \neq j \\
-\sum_{j=-M, j \neq i}^{M} C_{i j}^{(4)}
\end{array}\right.\right.
\end{aligned}
$$

(b) Regularized Shannon kernel (RSK) can also be used as a shape function such that the unknown $v$ and its derivatives can be approximated as a weighted linear sum of nodal values $v_{i},(i=-N: N)$ as follows : 
$\psi\left(x_{i}\right)=\sum_{j=-M}^{M}\left\langle\frac{\sin \left[\pi\left(x_{i}-x_{j}\right) / h_{x}\right]}{\pi\left(x_{i}-x_{j}\right) / h_{x}} e^{-\left(\frac{\left(x_{i}-x_{j}\right)^{2}}{2 \sigma^{2}}\right)}\right\rangle \psi\left(x_{j}\right), \quad(i=-N: N), \sigma=\left(r^{*} h_{x}\right)>0$

$\left.\frac{\partial v}{\partial x}\right|_{x=x_{i}}=\sum_{j=-M}^{M} C_{i j}^{(1)} v\left(x_{j}\right),\left.\quad \frac{\partial^{2} v}{\partial x^{2}}\right|_{x=x_{i}}=\sum_{j=-M}^{M} C_{i j}^{(2)} v\left(x_{j}\right), \quad(i=-N, N)$,

Similarly, one can approximate $\frac{\partial^{3} v}{\partial x^{3}}, \frac{\partial^{4} v}{\partial x^{4}}$ and calculate $C_{i j}^{(3)}, C_{i j}^{(4)}$

Where $\sigma$ is regularization parameter and $\mathrm{r}$ is a computational parameter. The weighting coefficients $C_{i j}^{(1)}, C_{i j}^{(2)}, C_{i j}^{(3)}$ and $C_{i j}^{(4)}$ can be defined as (Wei, 2001):

$$
\begin{aligned}
& C_{i j}^{(1)}=\left\{\begin{array}{ll}
\frac{(-1)^{i-j}}{h_{x}(i-j)} e^{-h_{x}{ }^{2}\left(\frac{(i-j)^{2}}{2 \sigma^{2}}\right)}, i \neq j \\
0 \quad i=j
\end{array}, C_{i j}^{(2)}= \begin{cases}\left(\frac{2(-1)^{i-j+1}}{h_{x}{ }^{2}(i-j)^{2}}+\frac{1}{\sigma^{2}}\right) e^{-h_{x}^{2}\left(\frac{(i-j)^{2}}{2 \sigma^{2}}\right)} & , i \neq j \\
-\frac{1}{\sigma^{2}-\frac{\pi^{2}}{3 h_{x}{ }^{2}}} & i=j\end{cases} \right. \\
& \mathrm{C}_{\mathrm{ij}}^{(3)}=\left\{\frac{(-1)^{\mathrm{i}-\mathrm{j}}}{h_{x}^{3}(\mathrm{i}-\mathrm{j})^{3}}\left(\frac{\pi^{2}}{h_{x}{ }^{3}(\mathrm{i}-\mathrm{j})}+\frac{6}{h_{x}{ }^{3}(\mathrm{i}-\mathrm{j})^{3}}+\frac{3}{h_{x}(\mathrm{i}-\mathrm{j}) \sigma^{2}}+\frac{3 h_{x}(\mathrm{i}-\mathrm{j})}{\sigma^{4}}\right)_{i=j}^{-h_{x}^{2}\left(\frac{(i-j)^{2}}{2 \sigma^{2}}\right)}, \quad i \neq j\right. \\
& \mathrm{C}_{\mathrm{ij}}^{(4)}=\left\{\begin{array}{l}
(-1)^{\mathrm{i}-\mathrm{j}}\left(\frac{4 \pi^{2}}{h_{x}{ }^{4}(\mathrm{i}-\mathrm{j})^{2}}+\frac{4 \pi^{2}}{h_{x}{ }^{2} \sigma^{2}}-\frac{24}{h_{x}{ }^{4}(\mathrm{i}-\mathrm{j})^{4}}-\frac{12}{h_{x}{ }^{2}(\mathrm{i}-\mathrm{j})^{2} \sigma^{2}}-\frac{4 h_{x}{ }^{2}(\mathrm{i}-\mathrm{j})^{2}}{\sigma^{6}}\right) e^{-h_{x}{ }^{2}\left(\frac{(i-j)^{2}}{2 \sigma^{2}}\right)}, \mathrm{i} \neq \mathrm{j} \\
\frac{3}{\sigma^{4}}+\frac{2 \pi^{2}}{h_{x}{ }^{2} \sigma^{2}}+\frac{\pi^{4}}{5 h_{x}{ }^{4}} \\
\mathrm{i}=\mathrm{j}
\end{array}\right.
\end{aligned}
$$

On suitable substitution from equations of weighting coefficients (36) into (14-17), the problem can be reduced to the following nonlinear Eigen-value problem:

$$
\begin{array}{r}
\mathrm{k}_{\mathrm{s}} \overline{\mathrm{A}}_{44}\left[\sum_{\mathrm{j}=1}^{\mathrm{N}} \mathrm{C}_{\mathrm{ij}}^{(2)} \mathrm{W}_{\mathrm{j}}+\eta \sum_{\mathrm{j}=1}^{\mathrm{N}} \mathrm{C}_{\mathrm{ij}}^{(1)} \Theta_{\mathrm{j}}\right]-\mathrm{k}_{\mathrm{s}}\left(\overline{\mathrm{E}}_{15} \sum_{\mathrm{j}=1}^{\mathrm{N}} \mathrm{C}_{\mathrm{ij}}^{(2)} \Phi_{\mathrm{j}}+\overline{\mathrm{Q}}_{15} \sum_{\mathrm{j}=1}^{\mathrm{N}} \mathrm{C}_{\mathrm{ij}}^{(2)} \Psi_{j}\right)+\left(\overline{\mathrm{N}}_{\mathrm{E}}+\overline{\mathrm{N}}_{\mathrm{T}}+\overline{\mathrm{N}}_{\mathrm{M}}+\overline{\mathrm{N}}_{\mathrm{P}}\right) \\
\left(\sum_{\mathrm{j}=1}^{\mathrm{N}} \mathrm{C}_{\mathrm{ij}}^{(2)} \mathrm{W}_{\mathrm{j}}-\mu^{2} \sum_{\mathrm{j}=1}^{\mathrm{N}} \mathrm{C}_{\mathrm{ij}}^{(4)} \mathrm{W}_{\mathrm{j}}\right)-\mathrm{k}_{1}\left(\sum_{\mathrm{j}=1}^{\mathrm{N}} \delta_{\mathrm{ij}} \mathrm{W}_{\mathrm{j}}-\mu^{2} \sum_{\mathrm{j}=1}^{\mathrm{N}} \mathrm{C}_{\mathrm{ij}}^{(2)} \mathrm{W}_{\mathrm{j}}\right)+\mathrm{k}_{2}\left(\sum_{\mathrm{j}=1}^{\mathrm{N}} \mathrm{C}_{\mathrm{ij}}^{(2)} \mathrm{W}_{\mathrm{j}}-\mu^{2} \sum_{\mathrm{j}=1}^{\mathrm{N}} \mathrm{C}_{\mathrm{ij}}^{(4)} \mathrm{W}_{\mathrm{j}}\right)+\mathrm{k}_{3} \sum_{\mathrm{j}=1}^{\mathrm{N}} \delta_{\mathrm{ij}} \mathrm{W}_{\mathrm{j}}^{3} \\
=-\overline{\mathrm{I}}_{0} \omega^{2}\left[\sum_{\mathrm{j}=1}^{\mathrm{N}} \delta_{\mathrm{ij}} \mathrm{W}_{\mathrm{j}}-\mu^{2} \sum_{\mathrm{j}=1}^{\mathrm{N}} \mathrm{C}_{\mathrm{ij}}^{(2)} \mathrm{W}_{\mathrm{j}}\right]
\end{array}
$$




$$
\begin{array}{r}
\bar{D}_{11} \sum_{j=1}^{N} C_{i j}^{(2)} \Theta_{j}-k_{s} \bar{A}_{44} \eta\left(\sum_{j=1}^{N} C_{i j}^{(1)} W_{j}+\eta \sum_{j=1}^{N} \delta_{i j} \Theta_{j}\right)+\left(\bar{Q}_{31}+k_{s} \bar{Q}_{15}\right) \eta \sum_{j=1}^{N} C_{i j}^{(1)} \Psi_{j}+\left(\bar{E}_{31}+k_{s} \bar{E}_{15}\right) \eta \sum_{j=1}^{N} C_{i j}^{(1)} \Phi_{j} \\
=-\bar{I}_{2} \omega^{2}\left[\underset{j=1}{N} \delta_{i j} \Theta_{j}-\mu^{2} \sum_{j=1}^{N} C_{i j}^{(2)} \Theta_{j}\right],
\end{array}
$$

$$
\overline{\mathrm{E}}_{31} \eta \sum_{j=1}^{\mathrm{N}} \mathrm{C}_{\mathrm{ij}}^{(1)} \Theta_{j}+\overline{\mathrm{E}}_{15}\left[\sum_{\mathrm{j}=1}^{\mathrm{N}} \mathrm{C}_{\mathrm{ij}}^{(2)} \mathrm{W}_{\mathrm{j}}+\eta \sum_{\mathrm{j}=1}^{\mathrm{N}} \mathrm{C}_{\mathrm{ij}}^{(1)} \Theta_{\mathrm{j}}\right]+\overline{\mathrm{X}}_{11} \sum_{\mathrm{j}=1}^{\mathrm{N}} \mathrm{C}_{\mathrm{ij}}^{(2)} \Phi_{\mathrm{j}}-\overline{\mathrm{X}}_{33} \eta^{2} \sum_{j=1}^{\mathrm{N}} \delta_{i j} \Phi_{j}+\overline{\mathrm{Y}}_{11} \sum_{j=1}^{\mathrm{N}} \mathrm{C}_{\mathrm{ij}}^{(2)} \Psi_{j}-\bar{Y}_{33} \eta^{2} \sum_{j=1}^{\mathrm{N}} \delta_{\mathrm{ij}} \Psi_{j}=0
$$

$\bar{Q}_{31} \eta \sum_{j=1}^{N} C_{i j}^{(1)} \Theta_{j}+\bar{Q}_{15}\left[\sum_{j=1}^{N} C_{i j}^{(2)} W_{j}+\eta \sum_{j=1}^{N} C_{i j}^{(1)} \Theta_{j}\right]+\bar{Y}_{11} \sum_{j=1}^{N} C_{i j}^{(2)} \Phi_{j}-$

$\bar{Y}_{33} \eta^{2} \sum_{j=1}^{N} \delta_{i j} \Phi_{j}+\bar{T}_{11} \sum_{j=1}^{N} C_{i j}^{(2)} \Psi_{j}-\bar{T}_{33} \eta^{2} \sum_{j=1}^{N} \delta_{i j} \Psi_{j}=0$

The boundary conditions (18-20) can also be approximated using three DQMs as:

$$
\begin{array}{lll}
W_{1}=\Theta_{1}=\Phi_{1}=\Psi_{1}=0, & \text { at } \zeta=0, \\
W_{N}=\Theta_{N}=\Phi_{N}=\Psi_{N}=0, & \text { at } \zeta=1,
\end{array}
$$

(2) Hinged (H):

$$
\begin{aligned}
& W_{1}=\Phi_{1}=\Psi_{1}=0 \\
& \bar{D}_{11} \sum_{j=1}^{N} C_{1 j}^{(1)} \Theta_{j}+\bar{E}_{31} \eta\left(\sum_{j=1}^{N} \delta_{1 j} \Phi_{j}+\sum_{j=1}^{N} \delta_{1 j} \Psi_{j}\right)-\mu^{2} \omega^{2}\left[\bar{I}_{2} \sum_{j=1}^{N} C_{1 j}^{(1)} \Theta_{j}+\bar{I}_{0} \eta \sum_{j=1}^{N} \delta_{1 j} W_{j}\right] \\
& -\mu^{2}\left(k_{1} \sum_{j=1}^{N} \delta_{1 j} W_{j}+k_{2} \eta \sum_{j=1}^{N} C_{1 j}^{(2)} W_{j}+\left(\overline{\mathrm{N}}_{\mathrm{T}}+\overline{\mathrm{N}}_{\mathrm{E}}+\overline{\mathrm{N}}_{P}+\overline{\mathrm{N}}_{M}\right) \eta \sum_{j=1}^{\mathrm{N}} \mathrm{C}_{1 j}^{(2)} W_{j}\right)=0, \quad \text { at } \zeta=0 \\
& W_{N}=\Phi_{N}=\Psi_{N}=0 \\
& \bar{D}_{11} \sum_{j=1}^{N} C_{N j}^{(1)} \Theta_{j}+\bar{E}_{31} \eta\left(\sum_{j=1}^{N} \delta_{N j} \Phi_{j}+\sum_{j=1}^{N} \delta_{N j} \Psi_{j}\right)-\mu^{2} \omega^{2}\left[\bar{I}_{2} \sum_{j=1}^{N} C_{N j}^{(1)} \Theta_{j}+\bar{I}_{0} \eta \sum_{j=1}^{N} \delta_{N j} W_{j}\right] \\
& -\mu^{2}\left(k_{1} \sum_{j=1}^{N} \delta_{N j} W_{j}+k k_{2} \eta \sum_{j=1}^{N} C_{N j}^{(2)} W_{j}+\left(\overline{\mathrm{N}}_{\mathrm{T}}+\overline{\mathrm{N}}_{\mathrm{E}}+\overline{\mathrm{N}}_{P}+\overline{\mathrm{N}}_{M}\right) \eta \sum_{j=1}^{\mathrm{N}} \mathrm{C}_{N j}^{(2)} W_{j}\right)=0, \quad \text { at } \zeta=N
\end{aligned}
$$

Then, using the iterative quadrature technique (Ragb et al., 2017) to obtain linear an Eigen-value problem as:

1- Firstly, solving the eqs. (35-38) as linear system 


$$
\begin{aligned}
& \mathrm{k}_{\mathrm{S}} \overline{\mathrm{A}}_{44}\left[\sum_{j=1}^{N} C_{i j}^{(2)} W_{j}+\eta \sum_{j=1}^{N} C_{i j}^{(1)} \Theta_{j}\right]-\mathrm{k}_{\mathrm{S}}\left(\overline{\mathrm{E}}_{15} \sum_{j=1}^{N} C_{i j}^{(2)} \Phi_{j}+\bar{Q}_{15} \sum_{j=1}^{N} C_{i j}^{(2)} \Psi_{j}\right)+\left(\overline{\mathrm{N}}_{\mathrm{E}}+\overline{\mathrm{N}}_{\mathrm{T}}+\overline{\mathrm{N}}_{M}+\overline{\mathrm{N}}_{P}\right) \\
& \left(\sum_{j=1}^{N} C_{i j}^{(2)} W_{j}-\mu^{2} \sum_{j=1}^{N} C_{i j}^{(4)} W_{j}\right)-k_{1}\left(\sum_{j=1}^{N} \delta_{i j} W_{j}-\mu^{2} \sum_{j=1}^{N} C_{i j}^{(2)} W_{j}\right)+k_{2}\left(\sum_{j=1}^{N} C_{i j}^{(2)} W_{j}-\mu^{2} \sum_{j=1}^{N} C_{i j}^{(4)} W_{j}\right)+ \\
& k_{3} \sum_{j=1}^{N} \delta_{i j} W_{j}=-\overline{\mathrm{I}}_{0} \omega^{2}\left[\sum_{j=1}^{N} \delta_{i j} W_{j}-\mu^{2} \sum_{j=1}^{N} C_{i j}^{(2)} W_{j}\right], \\
& \overline{\mathrm{D}}_{11} \sum_{j=1}^{N} C_{i j}^{(2)} \Theta_{j}-\mathrm{k}_{\mathrm{S}} \overline{\mathrm{A}}_{44} \eta\left(\sum_{j=1}^{N} C_{i j}^{(1)} W_{j}+\eta \sum_{j=1}^{N} \delta_{i j} \Theta_{j}\right)+\left(\bar{Q}_{31}+\mathrm{k}_{\mathrm{S}} \bar{Q}_{15}\right) \eta \sum_{j=1}^{N} C_{i j}^{(1)} \Psi_{j}+ \\
& \left(\bar{E}_{31}+\mathrm{k}_{\mathrm{S}} \overline{\mathrm{E}}_{15}\right) \eta \sum_{j=1}^{N} C_{i j}^{(1)} \Phi_{j}=-\overline{\mathrm{I}}_{2} \omega^{2}\left[\sum_{j=1}^{N} \delta_{i j} \Theta_{j}-\mu^{2} \sum_{j=1}^{N} C_{i j}^{(2)} \Theta_{j}\right],
\end{aligned}
$$

$\bar{E}_{31} \eta \sum_{j=1}^{N} C_{i j}^{(1)} \Theta_{j}+\overline{\mathrm{E}}_{15}\left[\sum_{j=1}^{N} C_{i j}^{(2)} W_{j}+\eta \sum_{j=1}^{N} C_{i j}^{(1)} \Theta_{j}\right]+\bar{X}_{11} \sum_{j=1}^{N} C_{i j}^{(2)} \Phi_{j}-$

$\overline{\mathrm{X}}_{33} \eta^{2} \sum_{j=1}^{N} \delta_{i j} \Phi_{j}+\bar{Y}_{11} \sum_{j=1}^{N} C_{i j}^{(2)} \Psi_{j}-\bar{Y}_{33} \eta^{2} \sum_{j=1}^{N} \delta_{i j} \Psi_{j}=0$

$$
\begin{aligned}
& \bar{Q}_{31} \eta \sum_{j=1}^{N} C_{i j}^{(1)} \Theta_{j}+\bar{Q}_{15}\left[\sum_{j=1}^{N} C_{i j}^{(2)} W_{j}+\eta \sum_{j=1}^{N} C_{i j}^{(1)} \Theta_{j}\right]+\bar{Y}_{11} \sum_{j=1}^{N} C_{i j}^{(2)} \Phi_{j}- \\
& \bar{Y}_{33} \eta^{2} \sum_{j=1}^{N} \delta_{i j} \Phi_{j}+\bar{T}_{11} \sum_{j=1}^{N} C_{i j}^{(2)} \Psi_{j}-\bar{T}_{33} \eta^{2} \sum_{j=1}^{N} \delta_{i j} \Psi_{j}=0
\end{aligned}
$$

2- Then we solve the following iterative system until obtain the convergence condition

$$
\begin{aligned}
& \left|\frac{W_{k+1}}{W_{k}}\right|<1 \text { where } k=0,1,2 \\
& \mathrm{k}_{\mathrm{S}} \overline{\mathrm{A}}_{44}\left[\sum_{j=1}^{N} C_{i j}^{(2)} W_{k+1 j}+\eta \sum_{j=1}^{N} C_{i j}^{(1)} \Theta_{j}\right]-\mathrm{k}_{\mathrm{S}}\left(\overline{\mathrm{E}}_{15} \sum_{j=1}^{N} C_{i j}^{(2)} \Phi_{j}+\bar{Q}_{15} \sum_{j=1}^{N} C_{i j}^{(2)} \Psi_{j}\right)+\left(\overline{\mathrm{N}}_{\mathrm{E}}+\overline{\mathrm{N}}_{\mathrm{T}}+\overline{\mathrm{N}}_{M}+\overline{\mathrm{N}}_{P}\right) \\
& \left(\sum_{j=1}^{N} C_{i j}^{(2)} W_{k+1 j}-\mu^{2} \sum_{j=1}^{N} C_{i j}^{(4)} W_{k+1 j}\right)-k_{1}\left(\sum_{j=1}^{N} \delta_{i j} W_{k+1 j}-\mu^{2} \sum_{j=1}^{N} C_{i j}^{(2)} W_{k+1 j}\right)+k_{2}\left(\sum_{j=1}^{N} C_{i j}^{(2)} W_{k+1 j}-\mu^{2} \sum_{j=1}^{N} C_{i j}^{(4)} W_{k+1 j}\right) \\
& +k_{3} \sum_{j=1}^{N} \delta_{i j} W_{k j}^{2} W_{k+1 j}=-\overline{\mathrm{I}}_{0} \omega^{2}\left[\sum_{j=1}^{N} \delta_{i j} W_{k+1 j}-\mu^{2} \sum_{j=1}^{N} C_{i j}^{(2)} W_{k+1 j}\right], \\
& \overline{\mathrm{D}}_{11} \sum_{j=1}^{N} C_{i j}^{(2)} \Theta_{j}-\mathrm{k}_{\mathrm{s}} \overline{\mathrm{A}}_{44} \eta\left(\sum_{j=1}^{N} C_{i j}^{(1)} W_{k+1 j}+\eta \sum_{j=1}^{N} \delta_{i j} \Theta_{j}\right)+\left(\bar{Q}_{31}+\mathrm{k}_{\mathrm{s}} \bar{Q}_{15}\right) \eta \sum_{j=1}^{N} C_{i j}^{(1)} \Psi_{j}+\left(\bar{E}_{31}+\mathrm{k}_{\mathrm{s}} \overline{\mathrm{E}}_{15}\right) \eta \sum_{j=1}^{N} C_{i j}^{(1)} \Phi_{j} \\
& =-\overline{\mathrm{I}}_{2} \omega^{2}\left[\sum_{j=1}^{N} \delta_{i j} \Theta_{j}-\mu^{2} \sum_{j=1}^{N} C_{i j}^{(2)} \Theta_{j}\right],
\end{aligned}
$$




$$
\begin{array}{r}
\bar{E}_{31} \eta \sum_{j=1}^{N} C_{i j}^{(1)} \Theta_{j}+\overline{\mathrm{E}}_{15}\left[\sum_{j=1}^{N} C_{i j}^{(2)} W_{k+1 j}+\eta \sum_{j=1}^{N} C_{i j}^{(1)} \Theta_{j}\right]+\overline{\mathrm{X}}_{11} \sum_{j=1}^{N} C_{i j}^{(2)} \Phi_{j}-\overline{\mathrm{X}}_{33} \eta^{2} \sum_{j=1}^{N} \delta_{i j} \Phi_{j}+\bar{Y}_{11} \sum_{j=1}^{N} C_{i j}^{(2)} \Psi_{j} \\
-\bar{Y}_{33} \eta^{2} \sum_{j=1}^{N} \delta_{i j} \Psi_{j}=0 \\
\bar{Q}_{31} \eta \sum_{j=1}^{N} C_{i j}^{(1)} \Theta_{j}+\bar{Q}_{15}\left[\sum_{j=1}^{N} C_{i j}^{(2)} W_{k+1 j}+\eta \sum_{j=1}^{N} C_{i j}^{(1)} \Theta_{j}\right]+\bar{Y}_{11} \sum_{j=1}^{N} C_{i j}^{(2)} \Phi_{j}-\bar{Y}_{33} \eta^{2} \sum_{j=1}^{N} \delta_{i j} \Phi_{j}+\bar{T}_{11} \sum_{j=1}^{N} C_{i j}^{(2)} \Psi_{j} \\
-\bar{T}_{33} \eta^{2} \sum_{j=1}^{N} \delta_{i j} \Psi_{j}=0
\end{array}
$$

\section{Numerical Results}

More convergence and efficiency of each one of the proposed schemes for vibration analysis of magneto-electrothermo-elastic nanobeams are demonstrated by the present numerical results. For all results, the boundary conditions (41-44) are augmented in the governing equations (37-40) and solved by iterative quadrature technique in eqs. (45-52). The computational characteristics of each scheme are adapted to reach accurate results with error of order $\leq 10^{-10}$. The obtained frequencies $\omega$ can be evaluated such as

$\omega=\Omega L \sqrt{\frac{\mathrm{I}_{0}}{\mathrm{~A}_{11}}}$ where $\Omega$ is the natural frequency of piezoelectric nanobeam

For the present results, material parameters for the composite are listed in Table (1).

Table 1. Material properties of PTZ-5H-COFe2O4 and BiTiO3-COFE2O4 composite materials (Shashidhar, S., \& Li. Y. J., 2005; Jandaghian, A. A., \& Rahmani, O., 2016a)

\begin{tabular}{lccc}
\hline \multicolumn{1}{c}{ Material properties } & & BiTiO3-COFe2O4 & PTZ-5H-COFe2O4 \\
\hline & $C_{11}$ & 226 & 206 \\
Elastic Constant(GPa) & $C_{12}$ & 125 & 126.25 \\
& $C_{13}$ & 124 & 127.3 \\
& $C_{44}$ & 44.2 & 34.3 \\
Piezoelectric Constant $\left(\mathrm{C} / \mathrm{m}^{2}\right)$ & $e_{31}$ & -2.2 & -3.25 \\
& $e_{33}$ & 9.3 & 8.5 \\
\hline Piezo magnetic Constants $(\mathrm{N} / \mathrm{Am})$ & $e_{15}$ & 5.8 & 7.555 \\
\hline & $\varepsilon_{31}$ & 5.64 & 6.5465 \\
\hline & & & 290.15 \\
\hline
\end{tabular}




\begin{tabular}{|c|c|c|c|}
\hline & $q_{33}$ & 349.9 & 349.9 \\
\hline & $q_{15}$ & 275 & 275 \\
\hline & $d_{11}$ & 5.367 & 16.5 \\
\hline Magnetoelectric $(\mathrm{Ns} / \mathrm{VC}) * 10^{-12}$ & $d_{33}$ & 2737.5 & 20.7 \\
\hline & $\mu_{11}$ & -297 & -297 \\
\hline Magnetic $\left(\mathrm{Ns}^{2} / \mathrm{C}^{2}\right)^{*} 10^{-6}$ & $\mu_{33}$ & 83.5 & 83.5 \\
\hline & $\lambda_{1}$ & 4.74 & 5 \\
\hline Thermal module $\left(\mathrm{N} / \mathrm{m}^{2} \mathrm{~K}\right) * 10^{5}$ & $\lambda_{3}$ & 4.53 & 4.85 \\
\hline Pyroelectric $(\mathrm{C} / \mathrm{N}) * 10^{-6}$ & $p_{3}$ & 25 & 25 \\
\hline Pyro-magnetic $(\mathrm{Nm} / \mathrm{AK}) * 10^{-6}$ & $\beta_{3}$ & 5.19 & 5.19 \\
\hline Density $\left(\mathrm{kg} / \mathrm{m}^{3}\right)$ & $\rho$ & 5550 & 6550 \\
\hline
\end{tabular}

For PDQM the problem is solved over a non-uniform grids, with Gauss - Chebyshev - Lobatto discretizations, such as (Chang, 2000):

$$
x_{i}=\frac{1}{2}\left[1-\cos \left(\frac{i-1}{N-1} \pi\right)\right],(i=1: N)
$$

Where the dimensions of the grid $(\mathrm{N})$ ranges from 3 to 15 . The obtained results agreed with previous analytical ones (Jandaghian, A. A., \& Rahmani, O., 2016a; Ke, L. L., \& Wang, Y. S., 2014) over 11 grid size, as shown in Table 2

Table 2. Comparison between the obtained normalized frequencies, due to PDQM, and the previous exact and numerical ones, for various grid sizes: clamped clamped METE nanobeam $\left(\Delta T=0, \mathrm{~V}_{0}=0, P_{0}=0, \mathrm{~A}_{0}=0, \mathrm{~L}=80 \mathrm{~nm}, \mathrm{~h}=10 \mathrm{~nm}, \mu=0, \mathrm{k}_{1}=\mathrm{k}_{2}=\mathrm{k}_{3}=0\right)$.

\begin{tabular}{|c|c|c|c|c|c|}
\hline Normalized frequencies & $\omega_{1}$ & $\omega_{2}$ & $\omega_{3}$ & $\omega_{4}$ & $\omega_{5}$ \\
\hline \multicolumn{6}{|l|}{ Grid size $N$} \\
\hline 3 & 14.5445 & 145.484 & 165.349 & ----- & ----- \\
\hline 5 & 7.6348 & 30.1832 & 44.1502 & 147.015 & 161.182 \\
\hline 7 & 7.644 & 19.0072 & 32.8765 & 74.905 & 88.464 \\
\hline 9 & 7.646 & 18.679 & 32.4585 & 50.13 & 64.8814 \\
\hline 11 & 7.646 & 18.679 & 32.4772 & 48.095 & 64.0633 \\
\hline 13 & 7.646 & 18.679 & 32.4765 & 48.006 & 64.191 \\
\hline
\end{tabular}




\begin{tabular}{|c|c|c|c|c|c|}
\hline $\begin{array}{l}\text { Exact results (Jandaghian, A. } \\
\text { A., \& Rahmani, O., 2016a) }\end{array}$ & 7.6473 & 18.6692 & 32.4618 & ------- & ------ \\
\hline $\begin{array}{c}\text { PDQM (Ke, L. L., \& Wang, Y. } \\
\text { S., 2014) N=15 }\end{array}$ & 7.6267 & 18.6229 & 32.3964 & ------- & ------- \\
\hline Execution time (sec) & & 0.15-- & r 11 non & form grid & \\
\hline
\end{tabular}

For SincDQ scheme, the problem is solved over a regular grids ranging from 3 to 15 . Table 3 shows convergence of the obtained results. They agreed with exact ones (Jandaghian, A. A., \& Rahmani, O., 2016a) over grid size $\geq 9$. Also, this table shows that execution time of SincDQ scheme is less than that of PDQM. Therefor, it is more efficient than PDQM for vibration analysis of METE nanobeam.

Table 3. Comparison between the obtained normalized frequencies, due to SINC DQM, and the previous exact and numerical ones, for various grid sizes: clamped clamped METE nanobeam $\left(\Delta T=0, \mathrm{~V}_{0}=0, P_{0}=0, \mathrm{~A}_{0}=0, \mathrm{~L}=80 \mathrm{~nm}, \mathrm{~h}=10 \mathrm{~nm}, \mu=0, \mathrm{k}_{1}=\mathrm{k}_{2}=\mathrm{k}_{3}=0\right)$.

\begin{tabular}{|c|c|c|c|c|c|}
\hline Normalized frequencies & $\omega_{1}$ & $\omega_{2}$ & $\omega_{3}$ & $\omega_{4}$ & $\omega_{5}$ \\
\hline \multicolumn{6}{|l|}{ Grid size $\mathrm{N}$} \\
\hline 3 & 19.512 & 40.542 & 75.254 & ------- & -------- \\
\hline 5 & 16.3278 & 37.1149 & 68.3338 & 152.781 & 178.53 \\
\hline 7 & 9.5236 & 25.457 & 40.2145 & 60.214 & 78.254 \\
\hline 9 & 7.6469 & 18.6977 & 32.5948 & 48.1074 & 64.802 \\
\hline 11 & 7.6469 & 18.6977 & 32.5948 & 48.1074 & 64.802 \\
\hline $\begin{array}{l}\text { Exact results (Jandaghian, } \\
\text { A. A., \& Rahmani, O., } \\
\text { 2016a) }\end{array}$ & 7.6473 & 18.6692 & 32.4618 & ------- & ------ \\
\hline $\begin{array}{l}\text { PDQM (Ke, L. L., \& Wang, } \\
\text { Y. S., 2014) } \quad \mathrm{N}=15\end{array}$ & 7.6267 & 18.6229 & 32.3964 & ------- & ------- \\
\hline Execution time (sec) & & 0.1200-- & over $9 \mathrm{u}$ & orm grid & \\
\hline
\end{tabular}

For DSCDQM scheme based on delta Lagrange kernel, the problem is also solved over a uniform grid ranging from 3 to 11 . The bandwidth $2 \mathrm{M}+1$ ranges from 3 to 9 . Table 4 shows the convergence of the obtained fundamental frequency which agreed with exact ones (Jandaghian, A. A., \& Rahmani, O., 2016a) over grid size $\geq 3$ and bandwidth $\geq 3$. Tables $(4,5)$ show that the execution time of DSCDQM-DLK is less than that of PDQM and SincDQM.

Table 4. Comparison between the normalized fundamental frequency by using DSCDQM-DLK, bandwidth (2M+1) and grid size $\mathrm{N}$ for clamped-clamped METE nanobeam $\left(\Delta T=0, \mathrm{~V}_{0}=0, P_{0}=0, \mathrm{~A}_{0}=0, \mathrm{~L}=80 \mathrm{~nm}, \mathrm{~h}=10 \mathrm{~nm}, \mu=0, \mathrm{k}_{1}=\mathrm{k}_{2}=\mathrm{k}_{3}=0\right)$

\begin{tabular}{ccccccc}
\hline \multicolumn{2}{c}{ Fundamental frequency } & \multicolumn{5}{c}{ DSCDQM-DLK } \\
\hline Bandwidth & $\mathrm{N}$ & 3 & 5 & 7 & 9 & 11 \\
\hline $2 \mathrm{M}+1=3$ & 7.6469 & 7.6469 & 7.6469 & 7.6469 & 7.6469 \\
$2 \mathrm{M}+1=5$ & 7.6469 & 7.6469 & 7.6469 & 7.6469 & 7.6469 \\
$2 \mathrm{M}+1=7$ & 7.6469 & 7.6469 & 7.6469 & 7.6469 & 7.6469 \\
$2 \mathrm{M}+1=9$ & 7.6469 & 7.6469 & 7.6469 & 7.6469 & 7.6469 \\
\hline Execution time $(\mathrm{sec})$ & & $0.09--$ & over & 3 & uniform grid \\
\hline
\end{tabular}


Table 5. Comparison between the obtained normalized frequencies, due to DSCDQM-DLK, and the previous exact and numerical ones, for various grid sizes: clamped clamped METE nanobeam $\left(\Delta T=0, \mathrm{~V}_{0}=0, P_{0}=0, \mathrm{~A}_{0}=0, \mathrm{~L}=80 \mathrm{~nm}, \mathrm{~h}=10 \mathrm{~nm}, \mu=0, \mathrm{k}_{1}=\mathrm{k}_{2}=\mathrm{k}_{3}=0\right)$.

\begin{tabular}{|c|c|c|c|c|c|}
\hline Normalized frequencies & $\omega_{1}$ & $\omega_{2}$ & $\omega_{3}$ & $\omega_{4}$ & $\omega_{5}$ \\
\hline \multicolumn{6}{|l|}{ Grid size $N$} \\
\hline 3 & 7.6469 & 18.6977 & 32.5948 & 48.1074 & 64.802 \\
\hline 5 & 7.6469 & 18.6977 & 32.5948 & 48.1074 & 64.802 \\
\hline 7 & 7.6469 & 18.6977 & 32.5948 & 48.1074 & 64.802 \\
\hline 9 & 7.6469 & 18.6977 & 32.5948 & 48.1074 & 64.802 \\
\hline $\begin{array}{l}\text { Exact results (Jandaghian, A. } \\
\text { A., \& Rahmani, O., 2016a) }\end{array}$ & 7.6473 & 18.6692 & 32.4618 & |------ & ------ \\
\hline $\begin{array}{c}\text { PDQM (Ke, L. L., \& Wang, } \\
\text { Y. S., 2014) N=15 }\end{array}$ & 7.6267 & 18.6229 & 32.3964 & ------ & ------- \\
\hline Execution time (sec) & & 0.09-- & 3 un & $\mathrm{n}$ grid & \\
\hline
\end{tabular}

For DSCDQM scheme based on regularized Shannon kernel (RSK), the problem is also solved over a uniform grid ranging from 3 to 9 . The bandwidth $2 \mathrm{M}+1$ ranges from 3 to 7 and the regularization parameter $\sigma=\mathrm{rh}_{\mathrm{x}}$ ranges from $1 h_{x}$ to $1.75 h_{x}$, where $h_{x}=1 / \mathrm{N}-1$. Table 6 shows the convergence of the obtained fundamental frequency to the exact and numerical ones (Jandaghian, A. A., \& Rahmani, O., 2016a; Ke, L. L., \& Wang, Y. S., 2014) over grid size $\geq 3$, bandwidth $\geq 3$ and regulization parameter $\sigma=1.75 \mathrm{~h}_{\mathrm{x}}$. Table 7 also ensures that the execution time of this scheme is the least. Therefore, DSCDQM-RSK scheme is the best choice among the examined quadrature schemes for vibration analysis of METE nanobeam.

Table 6. Comparison between the normalized fundamental frequency by using DSCDQM-RSK, bandwidth (2M+1) regularization parameter $\sigma$ and grid size $\mathrm{N}$ for clamped clamped METE nanobeam. $\left(\Delta T=0, \mathrm{~V}_{0}=0, P_{0}=0, \mathrm{~A}_{0}=0, \mathrm{~L}=80 \mathrm{~nm}, \mathrm{~h}=10 \mathrm{~nm}, \mu=0, \mathrm{k}_{1}=\mathrm{k}_{2}=\mathrm{k}_{3}=0\right)$

\begin{tabular}{ccccccc}
\hline $\begin{array}{c}\text { fundamental } \\
\text { frequency }\end{array}$ & $\begin{array}{c}\text { regularization } \\
\text { parameter }\end{array}$ & \multicolumn{5}{l}{ DSCDQM-RSK } \\
\hline $\mathrm{N}$ & $2 \mathrm{M}+1$ & $\sigma=0.5 * \mathrm{~h}_{\mathrm{x}}$ & $\sigma=1 * \mathrm{~h}_{\mathrm{x}}$ & $\sigma=1.25^{*} \mathrm{~h}_{\mathrm{x}}$ & $\sigma=1.5 * \mathrm{~h}_{\mathrm{x}}$ & $\sigma=1.75^{*} \mathrm{~h}_{\mathrm{x}}$ \\
\hline \multirow{3}{*}{3} & 3 & 9.5336 & 8.7325 & 8.3112 & 7.9568 & 7.6469 \\
& 5 & 9.5336 & 8.7325 & 8.3112 & 7.9568 & 7.6469 \\
& 7 & 9.5336 & 8.7325 & 8.3112 & 7.9568 & 7.6469 \\
5 & 3 & 9.5336 & 8.7325 & 8.3112 & 7.9568 & 7.6469 \\
& 5 & 9.5336 & 8.7325 & 8.3112 & 7.9568 & 7.6469 \\
7 & 7 & 9.5336 & 8.7325 & 8.3112 & 7.9568 & 7.6469 \\
& 3 & 9.5336 & 8.7325 & 8.3112 & 7.9568 & 7.6469 \\
9 & 5 & 9.5336 & 8.7325 & 8.3112 & 7.9568 & 7.6469 \\
& 7 & 9.5336 & 8.7325 & 8.3112 & 7.9568 & 7.6469 \\
& 3 & 9.5336 & 8.7325 & 8.3112 & 7.9568 & 7.6469 \\
& 5 & 9.5336 & 8.7325 & 8.3112 & 7.9568 & 7.6469 \\
& 7 & 9.5336 & 8.7325 & 8.3112 & 7.9568 & 7.6469 \\
\hline
\end{tabular}


Table 7. Comparison between the obtained normalized frequencies, due to DSCDQM-RSK and the previous exact and numerical ones, for various grid sizes:clamped clamped METE nanobeam $\left(\Delta T=0, \mathrm{~V}_{0}=0, P_{0}=0, \mathrm{~A}_{0}=0,2 \mathrm{M}+1=3, \mathrm{~L}=80 \mathrm{~nm}, \mathrm{~h}=10 \mathrm{~nm}, \mu=0, \mathrm{k}_{1}=\mathrm{k}_{2}=\mathrm{k}_{3}=0\right)$

\begin{tabular}{|c|c|c|c|c|c|}
\hline Normalized frequencies & $\omega_{1}$ & $\omega_{2}$ & $\omega_{3}$ & $\omega_{4}$ & $\omega_{5}$ \\
\hline \multicolumn{6}{|l|}{ Grid size $\mathrm{N}$} \\
\hline 3 & 7.6469 & 18.6977 & 32.5948 & 48.1074 & 64.802 \\
\hline 5 & 7.6469 & 18.6977 & 32.5948 & 48.1074 & 64.802 \\
\hline 7 & 7.6469 & 18.6977 & 32.5948 & 48.1074 & 64.802 \\
\hline 9 & 7.6469 & 18.6977 & 32.5948 & 48.1074 & 64.802 \\
\hline $\begin{array}{c}\text { Exact results (Jandaghian, A. A., \& } \\
\text { Rahmani, O., 2016a) }\end{array}$ & 7.6473 & 18.6692 & 32.4618 & ------ & ------ \\
\hline $\begin{array}{l}\text { PDQM ( Ke, L. L., \& Wang, Y. S., } \\
\text { 2014) } \mathrm{N}=15\end{array}$ & 7.6267 & 18.6229 & 32.3964 & ------- & ------ \\
\hline Execution time (sec) & & $0.053-$ & over & m grid & \\
\hline
\end{tabular}

Now, a parametric study is presented to show the influence of linear and nonlinear elastic foundations parameters, temperature change $\left(\Delta T{ }^{\circ} \mathrm{C}\right)$, external electric voltage $\left(\mathrm{V}_{0}\right)$, nonlocal parameter $(\mu)$, length-to-thickness ratio $(\mathrm{L} / \mathrm{h})$, axial forces $P_{0}(N)$, external magnetic potential $A_{0}(A)$, different boundary conditions and different materials on the values of natural frequencies and mode shapes. The parametric study is introduced over grid 3 nodes, bandwidth $\geq 3$ and regulization parameter $\sigma=1.75 \mathrm{~h}_{\mathrm{x}}$ by DSCDQM-RSK scheme.Tables (8-11) show that the fundamental frequency increases with increasing linear elastic foundation parameters. Also, the computations declare that the results do not affect significantly by nonlinear elastic foundation parameter $\mathrm{k}_{3}$. Also, the fundamental frequencies depend on the sign and magnitude of the magnetic potential and axial forces.

Figures $(2,3,7)$ and tables (10-11) show that the fundamental frequency decrease with increasing temperature change $\left(\Delta T{ }^{\circ} \mathrm{C}\right)$, external electric voltage $\left(\mathrm{V}_{0}\right)$, nonlocal parameter $(\mu)$ and length-to-thickness ratio $(\mathrm{L} / \mathrm{h})$.Also, Figures (4-6) and tables (8-9) show that the fundamental frequency increase with increasing axial forces $P_{0}(N)$ and external magnetic potential $A_{0}(A)$. As well as, Figures (8-11) show the first three normalized mode shapes $\mathrm{W}$ and electrical potential $\phi$ with time. These figures show that the amplitudes of displacement $\mathrm{W}$ and electrical potential $\phi$ increase with increasing linear and nonlinear elastic foundation parameters. From all figures, it is found that the METE nanobeam is insensitive to the temperature change while the axial forces, external electric and magnetic potential has the greatest effect on the natural frequencies. Also, Figures (3-4) show that the effect of the external electric potential is opposite to that of the external magnetic potential. Furthermore, Figures (2-11) show that the fundamental frequency, normalized amplitude $\mathrm{W}$ and normalized electrical potential $\phi$ for BiTiO3-COFe2O4 material is higher than PTZ-5H-COFe2O4 material. For all tables the nanobeam made of $\mathrm{BiTiO} 3-\mathrm{COFe} 2 \mathrm{O} 4$. 
Table 8. Comparison between the normalized frequencies $\omega$ and elastic foundation parameters, for various axial force $P_{0}(N)$ clamped clamped METE nanobeam $\left(\mathrm{A}_{0}=0, \Delta T=0, \mathrm{~V}_{0}=0, \mathrm{~L}=60 \mathrm{~nm}, \mathrm{~h}=10 \mathrm{~nm}, \mu=0.1\right)$

\begin{tabular}{|c|c|c|c|c|c|c|c|c|c|c|c|c|}
\hline \multicolumn{3}{|c|}{ Axial forces } & \multicolumn{2}{|c|}{$P_{0}=-1.5$} & \multicolumn{2}{|c|}{$P_{0}=-1$} & \multicolumn{2}{|c|}{$P_{0}=0$} & \multicolumn{2}{|c|}{$P_{0}=1$} & \multicolumn{2}{|c|}{$P_{0}=1.5$} \\
\hline \multicolumn{3}{|c|}{ Normalized frequencies } & $\omega_{1}$ & $\omega_{2}$ & $\omega_{1}$ & $\omega_{2}$ & $\omega_{1}$ & $\omega_{2}$ & $\omega_{1}$ & $\omega_{2}$ & $\omega_{1}$ & $\omega_{2}$ \\
\hline \multicolumn{3}{|c|}{ Elastic Parameters } & & & & & & & & & & \\
\hline \multirow[t]{8}{*}{$\mathrm{k}_{3}$} & $\mathrm{k}_{2}$ & $\mathrm{k}_{1}$ & & & & & & & & & & \\
\hline & & 0 & 11.745 & 24.203 & 11.781 & 24.264 & 11.85 & 24.383 & 11.919 & 24.502 & 11.954 & 24.561 \\
\hline & & 5 & 11.749 & 24.2055 & 11.784 & 24.266 & 11854 & 24.385 & 11.923 & 24.504 & 11.958 & 24.563 \\
\hline & 0 & 10 & 11.753 & 24.207 & 11.788 & 24.267 & 11.858 & 24.387 & 11.927 & 24.506 & 11.961 & 24.565 \\
\hline & & 15 & 11.757 & 24.2091 & 11.792 & 24.269 & 11.862 & 24.389 & 11.931 & 24.507 & 11.965 & 24.567 \\
\hline & & 25 & 11.765 & 24.213 & 11.8 & 24.273 & 11.869 & 24.392 & 11.938 & 24.511 & 11.973 & 24.57 \\
\hline & & 0 & 11.752 & 24.2147 & 11.787 & 24.275 & 11.857 & 24.394 & 11.926 & 24.513 & 11.96 & 24.572 \\
\hline & & 5 & 11.756 & 24.5165 & 11.791 & 24.277 & 11.861 & 24.396 & 11.93 & 24.515 & 11.964 & 24.574 \\
\hline \multirow[t]{5}{*}{0.025} & 0.025 & 10 & 11.76 & 24.2184 & 11.795 & 24.278 & 11.865 & 24.398 & 11.934 & 24.517 & 11.968 & 24.576 \\
\hline & & 15 & 11.764 & 24.22 & 11.799 & 24.28 & 11.868 & 24.4 & 11.937 & 24.518 & 11.972 & 24.577 \\
\hline & & 25 & 11.772 & 24.224 & 11.807 & 24.284 & 11.876 & 24.403 & 11.945 & 24.522 & 11.979 & 24.581 \\
\hline & & 0 & 11.76 & 24.226 & 11.794 & 24.286 & 11.864 & 24.405 & 11.933 & 24.524 & 11.967 & 24.583 \\
\hline & & 5 & 11.763 & 24.2276 & 11.798 & 24.288 & 11.867 & 24.407 & 11.936 & 24.526 & 11.971 & 24.585 \\
\hline \multirow[t]{5}{*}{0.05} & 0.05 & 10 & 11.767 & 24.229 & 11.802 & 24.289 & 11.871 & 24.409 & 11.94 & 24.527 & 11.975 & 24.586 \\
\hline & & 15 & 11.771 & 24.231 & 11.806 & 24.291 & 11.875 & 24.411 & 11.944 & 24.529 & 11.978 & 24.588 \\
\hline & & 25 & 11.778 & 24.235 & 11.813 & 24.295 & 11.883 & 24.414 & 11.952 & 24.533 & 11.986 & 24.592 \\
\hline & & 0 & 11.772 & 24.248 & 11.807 & 24.308 & 11.877 & 24.427 & 11.946 & 24.546 & 11.98 & 24.605 \\
\hline & & 5 & 11.776 & 24.25 & 11.811 & 24.31 & 11.881 & 24.429 & 11.95 & 24.547 & 11.984 & 24.606 \\
\hline \multirow[t]{5}{*}{0.1} & 0.1 & 10 & 11.78 & 24.252 & 11.815 & 24.312 & 11.884 & 24.431 & 11.953 & 24.549 & 11.988 & 24.608 \\
\hline & & 15 & 11.784 & 24.253 & 11.819 & 24.313 & 11.888 & 24.433 & 11.957 & 24.551 & 11.991 & 24.610 \\
\hline & & 25 & 11.792 & 24.257 & 11.627 & 24.317 & 11.896 & 24.436 & 11.965 & 24.555 & 11.999 & 24.614 \\
\hline & & 0 & 11.786 & 24.27 & 11.821 & 24.33 & 11.89 & 24.449 & 11.959 & 24.567 & 11.993 & 24.626 \\
\hline & & 5 & 11.79 & 24.272 & 11.825 & 24.332 & 11.894 & 24.451 & 11.963 & 24.569 & 11.997 & 24.628 \\
\hline \multirow[t]{3}{*}{0.15} & 0.15 & 10 & 11.793 & 24.274 & 11.828 & 24.334 & 11.898 & 24.453 & 11.966 & 24.571 & 12.001 & 24.63 \\
\hline & & 15 & 11.797 & 24.276 & 11.832 & 24.335 & 11.902 & 24.454 & 11.97 & 24.573 & 12.004 & 24.632 \\
\hline & & 25 & 11.81 & 24.279 & 11.84 & 24.339 & 11.909 & 24.458 & 11.978 & 24.576 & 12.012 & 24.635 \\
\hline
\end{tabular}


Table 9. Comparison between the normalized frequencies $\omega$ and elastic foundation parameters, for various external magnetic potential $A_{0}(A)$ : clamped -clamped METE nanobeam

$\left(P_{0}=0, \Delta T=0, \mathrm{~V}_{0}=0, \mathrm{~L}=60 \mathrm{~nm}, \mathrm{~h}=10 \mathrm{~nm}, \mu=0.1\right)$.

\begin{tabular}{|c|c|c|c|c|c|c|c|c|c|c|c|c|}
\hline \multirow{2}{*}{\multicolumn{3}{|c|}{$\begin{array}{l}\text { Magnetic potential } \\
\text { Normalized Frequencies }\end{array}$}} & \multicolumn{2}{|c|}{$A_{0}=-0.02$} & \multicolumn{2}{|c|}{$A_{0}=-0.01$} & \multicolumn{2}{|c|}{$A_{0}=0$} & \multicolumn{2}{|c|}{$A_{0}=0.01$} & \multicolumn{2}{|c|}{$A_{0}=0.02$} \\
\hline & & & $\omega_{1}$ & $\omega_{2}$ & $\omega_{1}$ & $\omega_{2}$ & $\omega_{1}$ & $\omega_{2}$ & $\omega_{1}$ & $\omega_{2}$ & $\omega_{1}$ & $\omega_{2}$ \\
\hline \multicolumn{13}{|c|}{ Elastic Parameters } \\
\hline $\mathrm{k}_{3}$ & $\mathrm{k}_{2}$ & $\mathrm{k}_{1}$ & & & & & & & & & & \\
\hline \multirow{5}{*}{0} & \multirow{5}{*}{0} & 0 & 11.599 & 23.953 & 11.726 & 24.169 & 11.85 & 24.383 & 11.973 & 24.595 & 12.095 & 24.804 \\
\hline & & 5 & 11.603 & 23.955 & 11.73 & 24.171 & 11.854 & 24.385 & 11.977 & 24.597 & 12.099 & 24.806 \\
\hline & & 10 & 11.607 & 23.957 & 11.733 & 24.173 & 11.858 & 24.387 & 11.981 & 24.598 & 12.102 & 24.808 \\
\hline & & 15 & 11.611 & 23.959 & 11.737 & 24.175 & 11.862 & 24.389 & 11.985 & 24.60 & 12.106 & 24.809 \\
\hline & & 25 & 11.619 & 23.963 & 11.745 & 24.179 & 11.869 & 24.392 & 11.992 & 24.604 & 12.113 & 24.813 \\
\hline \multirow{5}{*}{0.025} & \multirow{5}{*}{0.025} & 0 & 11.606 & 23.965 & 11.732 & 24.181 & 11.857 & 24.394 & 11.98 & 24.606 & 12.101 & 24.815 \\
\hline & & 5 & 11.61 & 23.967 & 11.736 & 24.182 & 11.861 & 24.396 & 11.984 & 24.607 & 12.105 & 24.817 \\
\hline & & 10 & 11.614 & 23.968 & 11.74 & 24.184 & 11.865 & 24.398 & 11.987 & 24.609 & 12.109 & 24.818 \\
\hline & & 15 & 11.618 & 23.97 & 11.744 & 24.186 & 11.868 & 24.4 & 11.991 & 24.611 & 12.112 & 24.82 \\
\hline & & 25 & 11.626 & 23.974 & 11.752 & 24.19 & 11.876 & 24.403 & 11.999 & 24.615 & 12.12 & 24.824 \\
\hline \multirow{5}{*}{0.05} & \multirow{5}{*}{0.05} & 0 & 11.613 & 23.976 & 11.739 & 24.192 & 11.864 & 24.405 & 11.986 & 24.616 & 12.108 & 24.826 \\
\hline & & 5 & 11.617 & 23.978 & 11.743 & 24.193 & 11.867 & 24.407 & 11.99 & 24.618 & 12.111 & 24.827 \\
\hline & & 10 & 11.621 & 23.98 & 11.747 & 24.195 & 11.871 & 24.409 & 11.994 & 24.62 & 12.115 & 24.829 \\
\hline & & 15 & 11.625 & 23.981 & 11.751 & 24.197 & 11.875 & 24.411 & 11.998 & 24.622 & 12.119 & 24.831 \\
\hline & & 25 & 11.632 & 23.985 & 11.758 & 24.201 & 11.883 & 24.414 & 12.005 & 24.625 & 12.126 & 24.835 \\
\hline \multirow{5}{*}{0.1} & \multirow{5}{*}{0.1} & 0 & 11.626 & 23.998 & 11.752 & 24.214 & 11.877 & 24.427 & 12 & 24.638 & 12.121 & 24.847 \\
\hline & & 5 & 11.630 & 24.00 & 11.756 & 24.516 & 11.881 & 24.429 & 12.003 & 24.64 & 12.124 & 24.849 \\
\hline & & 10 & 11.634 & 24.002 & 11.76 & 24.217 & 11.884 & 24.431 & 12.007 & 24.642 & 12.128 & 24.851 \\
\hline & & 15 & 11.638 & 24.004 & 11.764 & 24.219 & 11.888 & 24.432 & 12.011 & 24.644 & 12.132 & 24.852 \\
\hline & & 25 & 11.646 & 24.007 & 11.772 & 24.223 & 11.896 & 24.436 & 12.018 & 24.647 & 12.139 & 24.856 \\
\hline \multirow{5}{*}{0.15} & \multirow{5}{*}{0.15} & 0 & 11.64 & 24.021 & 11.766 & 24.236 & 11.89 & 24.449 & 12.013 & 24.66 & 12.134 & 24.869 \\
\hline & & 5 & 11.644 & 24.022 & 11.77 & 24.238 & 11.894 & 24.451 & 12.016 & 24.662 & 12.137 & 24.87 \\
\hline & & 10 & 11.648 & 24.024 & 11.774 & 24.24 & 11.898 & 24.453 & 12.02 & 24.663 & 12.141 & 24.872 \\
\hline & & 15 & 11.652 & 24.026 & 11.777 & 24.241 & 11.901 & 24.454 & 12.024 & 24.665 & 12.145 & 24.874 \\
\hline & & 25 & 11.66 & 24.03 & 11.785 & 24.245 & 11.909 & 24.458 & 12.031 & 24.669 & 12.152 & 24.878 \\
\hline
\end{tabular}


Table 10. Comparison between the normalized frequencies, boundary conditions and nonlocal parameter $(\mu)$ for METE nanobeam $\left(P_{0}=0, \mathrm{~A}_{0}=0.02, \Delta T=0, \mathrm{~V}_{0}=0, \mathrm{~L}=80 \mathrm{~nm}, \mathrm{~L} / \mathrm{h}=8, \mathrm{k}_{1}=10, \mathrm{k}_{2}=0.025, \mathrm{k}_{3}=0.15\right)$.

\begin{tabular}{ccccccc}
\hline & & \multicolumn{5}{c}{ Normalized frequencies } \\
\cline { 2 - 6 } B.C & $\mu$ & $\omega_{1}$ & $\omega_{2}$ & $\omega_{3}$ & $\omega_{4}$ & $\omega_{5}$ \\
& 0 & 5.8127 & 16.5508 & 30.5288 & 46.4423 & 63.1618 \\
& 0.05 & 5.7455 & 15.8011 & 27.6732 & 39.4747 & 49.9575 \\
$\mathrm{CH}$ & 0.1 & 5.5596 & 14.0742 & 22.4378 & 29.383 & 34.673 \\
& 0.15 & 5.2935 & 12.2016 & 18.0756 & 22.5677 & 25.8567 \\
& 0.2 & 4.9905 & 10.5937 & 14.9913 & 18.2813 & 20.6293 \\
& 0.3 & 4.3971 & 8.3366 & 11.3117 & 13.4537 & 14.6772 \\
\hline & 0 & 7.8228 & 18.9123 & 32.7410 & 48.2897 & 64.5063 \\
& 0.05 & 7.7286 & 18.0251 & 29.6487 & 41.0333 & 51.045 \\
$\mathrm{CC}$ & 0.1 & 7.4687 & 15.9906 & 24.0051 & 30.5441 & 35.4677 \\
& 0.15 & 7.0987 & 13.8005 & 19.3378 & 23.4804 & 26.4795 \\
& 0.2 & 6.6802 & 11.9355 & 16.0648 & 19.0330 & 21.0858 \\
& 0.3 & 5.8704 & 9.3446 & 12.2038 & 13.922 & 14.8882 \\
\hline & 0 & 4.0711 & 14.1202 & 28.1803 & 44.4842 & 61.727 \\
& 0.05 & 4.0296 & 13.5050 & 25.2715 & 37.814 & 48.785 \\
$\mathrm{HH}$ & 0.1 & 3.9141 & 12.0759 & 20.7633 & 28.1311 & 33.799 \\
& 0.15 & 3.7468 & 10.506 & 16.7268 & 21.5699 & 25.1501 \\
& 0.2 & 3.5532 & 9.1397 & 13.8537 & 17.4392 & 20.0658 \\
\hline
\end{tabular}

Table 11. Comparison between the normalized frequencies, boundary conditions and length-to-thickness ratio $(\mathrm{L} / \mathrm{h})$ for METE nanobeam $\left(P_{0}=0, \mathrm{~A}_{0}=0.02, \Delta T=0, \mathrm{~V}_{0}=0, \mathrm{~h}=2 \mathrm{~nm}, \mu=0.1, \mathrm{k}_{1}=10, \mathrm{k}_{2}=0.025, \mathrm{k}_{3}=0.15\right)$

\begin{tabular}{ccccccc}
\hline & & \multicolumn{5}{c}{ Normalized frequencies } \\
\cline { 3 - 7 } B.C & $\mathrm{L} / \mathrm{h}$ & $\omega_{1}$ & $\omega_{2}$ & $\omega_{3}$ & $\omega_{4}$ & $\omega_{5}$ \\
\hline & 6 & 65.3075 & 132.3290 & 193.2852 & 240.0726 & 274.7364 \\
& 8 & 41.9400 & 87.6475 & 130.6966 & 166.538 & 193.0549 \\
$\mathrm{CC}$ & 12 & 22.8307 & 48.2839 & 73.7153 & 96.2304 & 114.8452 \\
& 16 & 15.396 & 32.2589 & 49.1744 & 65.2192 & 78.5352 \\
& 20 & 11.8894 & 24.2939 & 37.0331 & 48.9753 & 59.8226 \\
& 30 & 8.1887 & 16.0752 & 24.0178 & 31.6047 & 38.7174 \\
\hline & 6 & 51.369 & 120.3003 & 184.3095 & 234.5509 & 271.6168 \\
& 8 & 32.9497 & 78.0707 & 122.6264 & 160.4316 & 189.2995 \\
& 12 & 18.3886 & 42.4297 & 67.8774 & 91.1052 & 110.9483 \\
& 16 & 12.6912 & 28.4750 & 44.9936 & 61.2217 & 75.2852 \\
& 20 & 10.1075 & 21.495 & 33.8316 & 45.7028 & 56.9546 \\
& 30 & 7.244 & 14.4196 & 22.0180 & 29.4070 & 36.6993 \\
\hline \multirow{4}{*}{$\mathrm{CH}$} & 6 & 39.045 & 107.2164 & 174.6817 & 228.4481 & 268.5102 \\
& 8 & 25.4156 & 68.2893 & 114.1099 & 153.9207 & 185.1885 \\
& 12 & 14.8655 & 36.8893 & 62.0062 & 85.8467 & 106.6789 \\
& 16 & 10.7651 & 24.8817 & 41.0727 & 57.1300 & 71.8685 \\
& 20 & 8.6841 & 19.0506 & 30.7592 & 42.589 & 53.8329 \\
& 30 & 6.8041 & 13.008 & 20.1487 & 27.4042 & 34.5047 \\
\hline
\end{tabular}



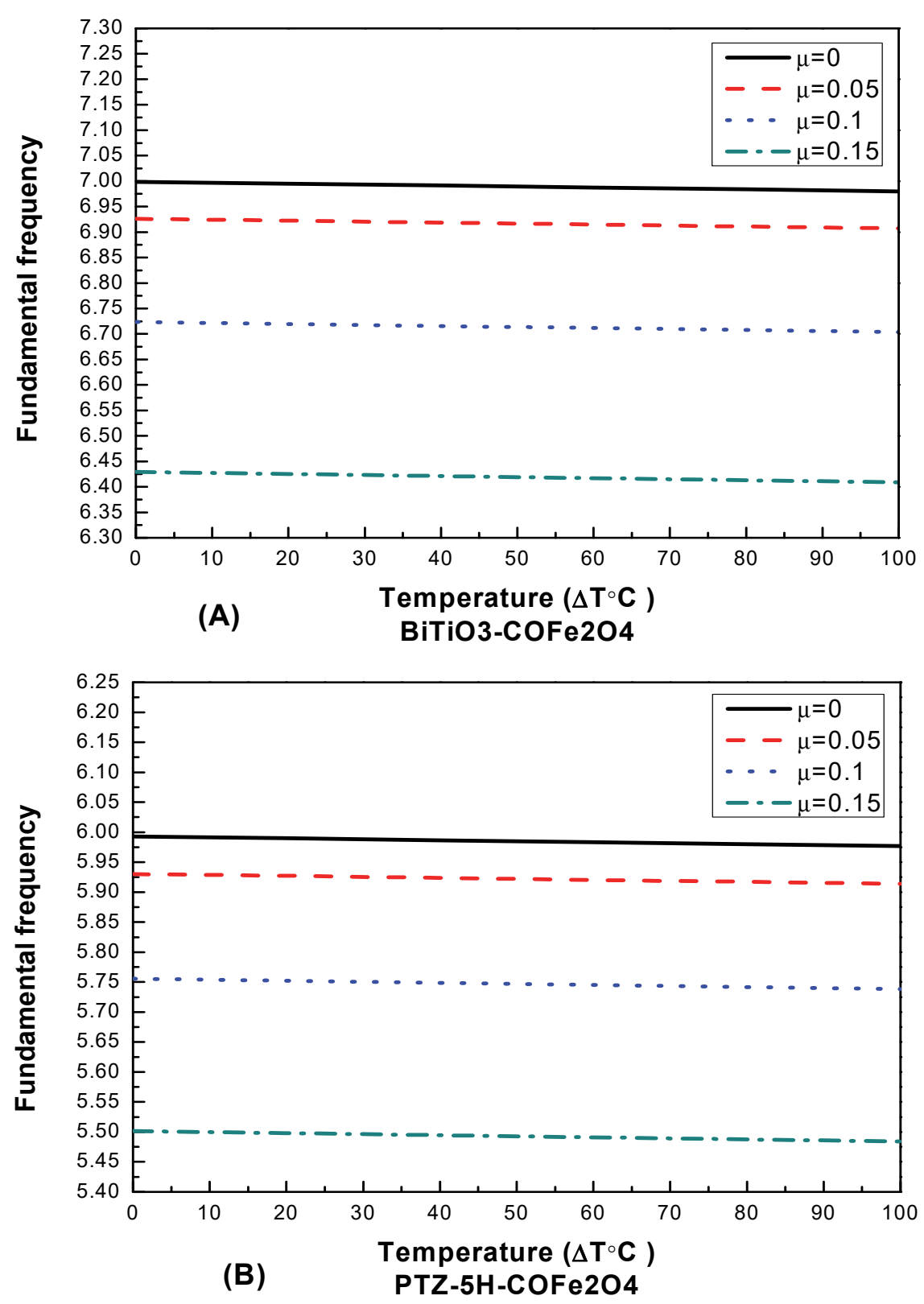

Figure 2. Variation of fundamental frequency with temperature $\left(\Delta T{ }^{\circ} \mathrm{C}\right)$, nonlocal parameter $(\mu)$ and different materials: (A) BiTiO3-COFe2O4; (B) PTZ-5H-COFe2O4 for Hinged-Hinged METE nanobeam.

$$
\left(P_{0}=1.5, \mathrm{~A}_{0}=0.02, \mathrm{~V}_{0}=0, \mathrm{~h}=10, \mathrm{~L} / \mathrm{h}=6, \mathrm{k}_{1}=25, \mathrm{k}_{2}=0.05, \mathrm{k}_{3}=0.15\right)
$$




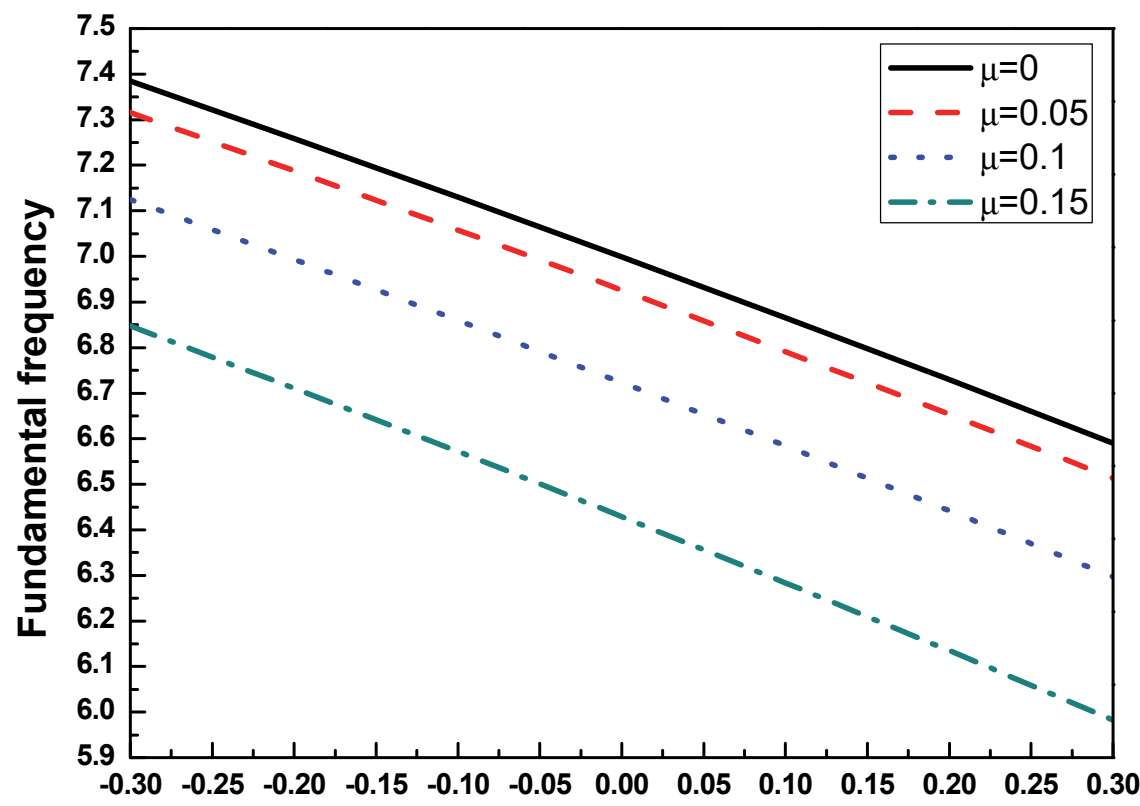

(A)

\section{External electric voltage}

BiTiO3-COFe2O4

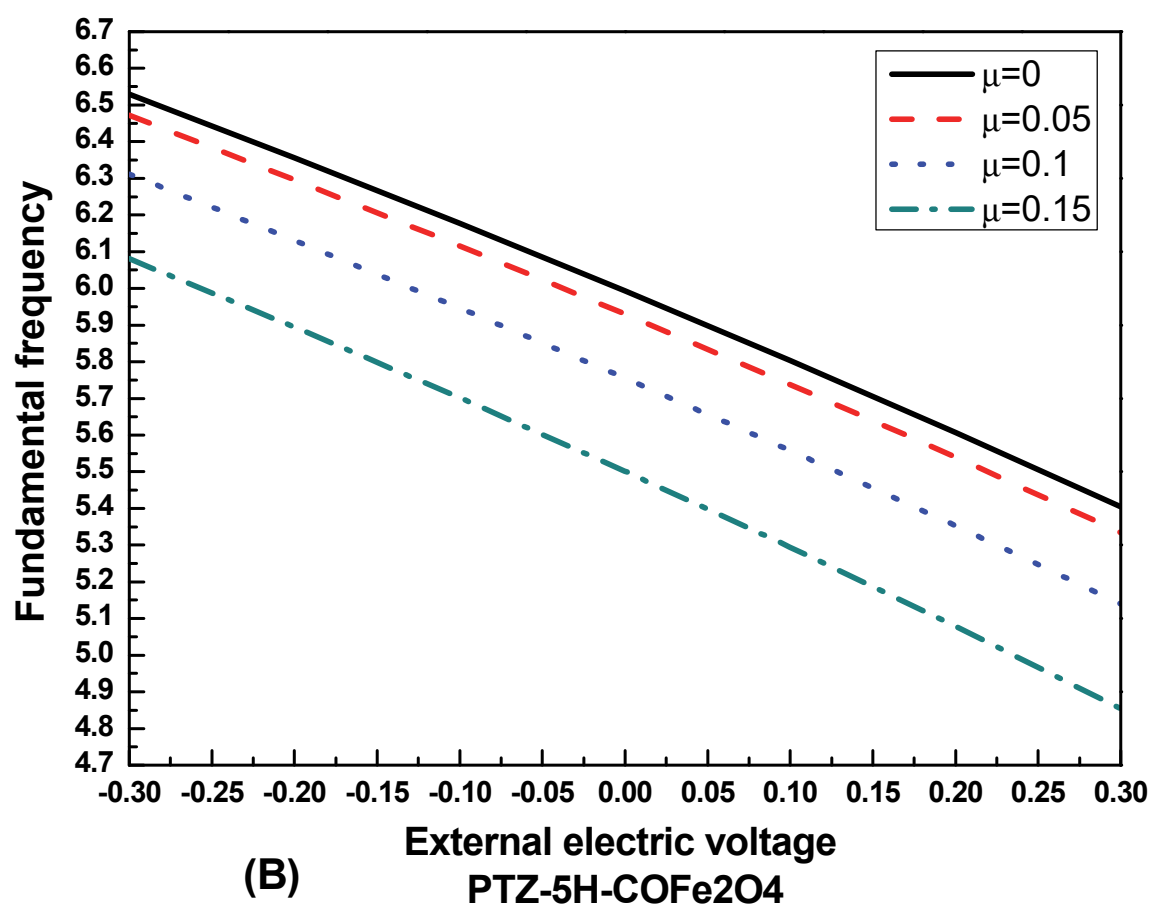

Figure 3. Variation of fundamental frequency with external electric voltage $\left(\mathrm{V}_{0}\right)$, nonlocal parameter $(\mu)$ and different materials: (A) BiTiO3-COFe2O4; (B) PTZ-5H-COFe2O4 for Hinged-Hinged METE nanobeam.

$$
\left(P_{0}=1.5, \mathrm{~A}_{0}=0.02, \Delta T=0, \mathrm{~h}=10, \mathrm{~L} / \mathrm{h}=6, \mathrm{k}_{1}=25, \mathrm{k}_{2}=0.05, \mathrm{k}_{3}=0.15\right)
$$



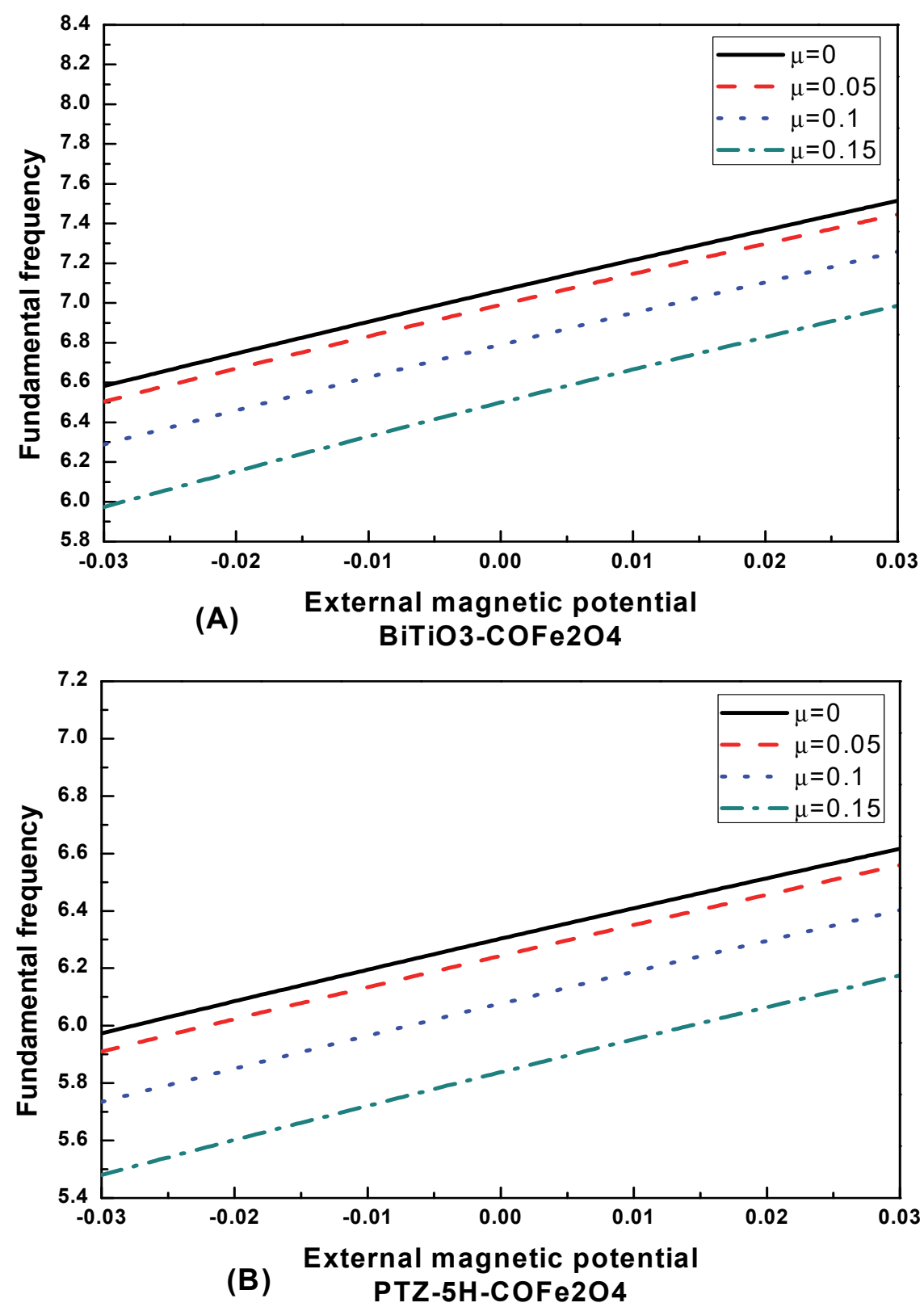

Figure 4. Variation of fundamental frequency with external magnetic potential $\left(A_{0}\right)$, nonlocal parameter $(\mu)$ and different materials: (A) BiTiO3-COFe2O4; (B) PTZ-5H-COFe2O4 for Hinged-Hinged METE nanobeam. $\left(P_{0}=1.5, \mathrm{~V}_{0}=-0.3, \Delta T=100, \mathrm{~h}=10, \mathrm{~L} / \mathrm{h}=6, \mathrm{k}_{1}=25, \mathrm{k}_{2}=0.05, \mathrm{k}_{3}=0.15\right)$ 

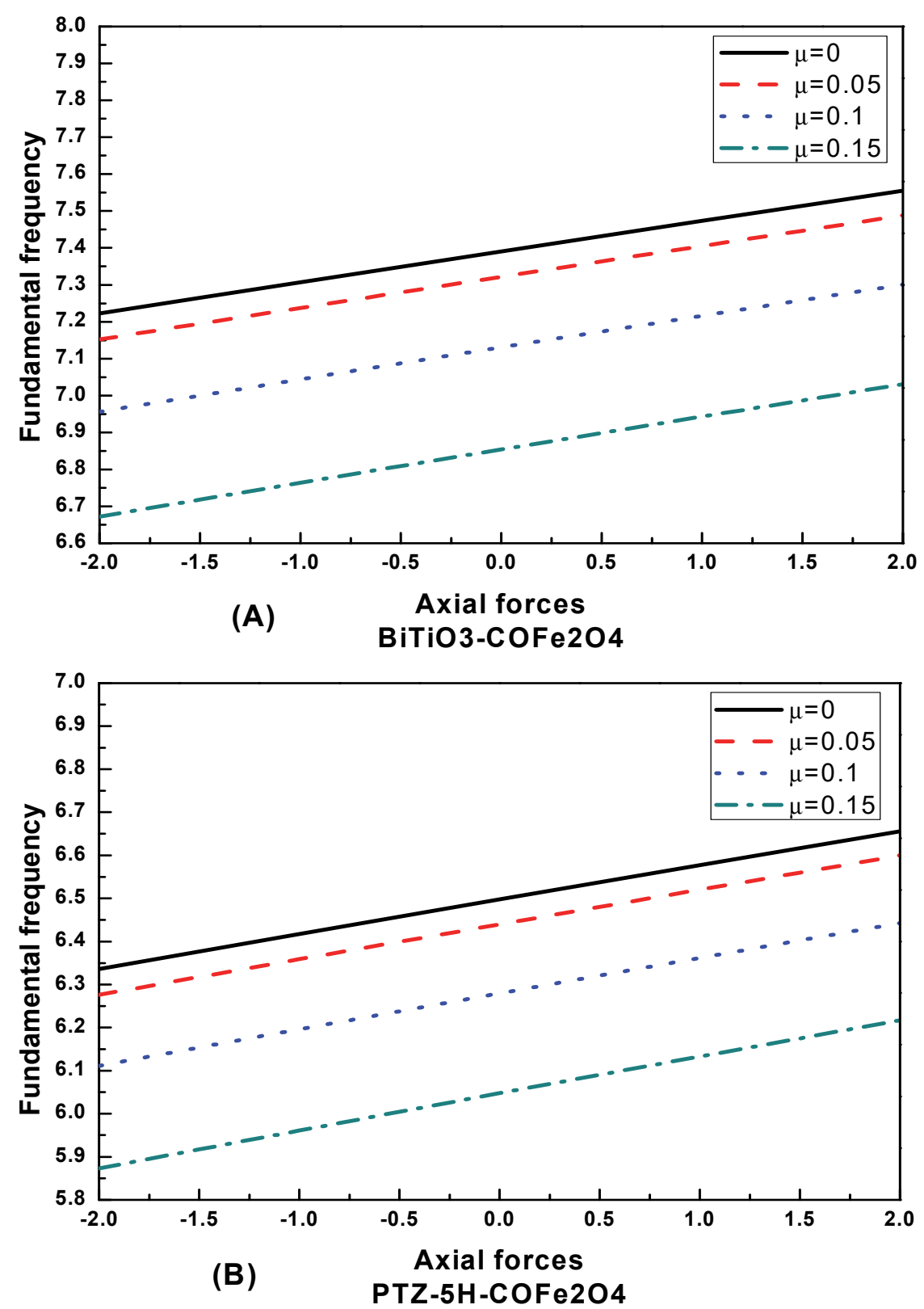

Figure 5. Variation of fundamental frequency with axial forces $\left(P_{0}\right)$, nonlocal parameter $(\mu)$ and different materials: (A) BiTiO3-COFe2O4; (B) PTZ-5H-COFe2O4 for Hinged-Hinged METE nanobeam.

$$
\left(V_{0}=-0.3, \mathrm{~A}_{0}=0.03, \Delta T=100, \mathrm{~h}=10, \mathrm{~L} / \mathrm{h}=6, \mathrm{k}_{1}=25, \mathrm{k}_{2}=0.05, \mathrm{k}_{3}=0.15\right)
$$



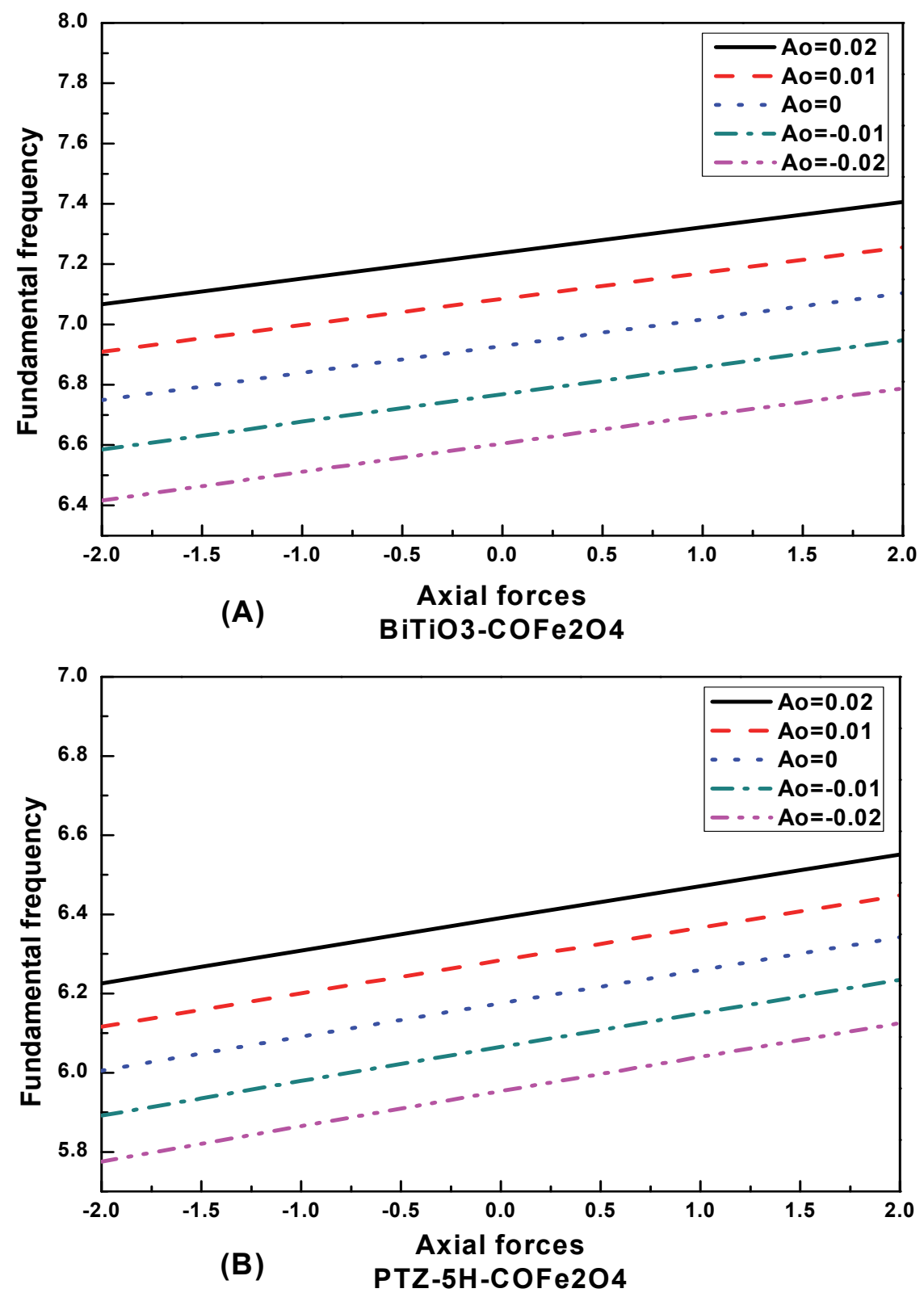

Figure 6. Variation of fundamental frequency with axial forces $\left(P_{0}\right)$, external magnetic potential $\left(A_{0}\right)$ and different materials: (A) BiTiO3-COFe2O4; (B) PTZ-5H-COFe2O4 for Hinged-Hinged METE nanobeam.

$$
\left(V_{0}=-0.3, \Delta T=100, \mu=0.01, \mathrm{~h}=10, \mathrm{~L} / \mathrm{h}=6, \mathrm{k}_{1}=25, \mathrm{k}_{2}=0.05, \mathrm{k}_{3}=0.15\right)
$$




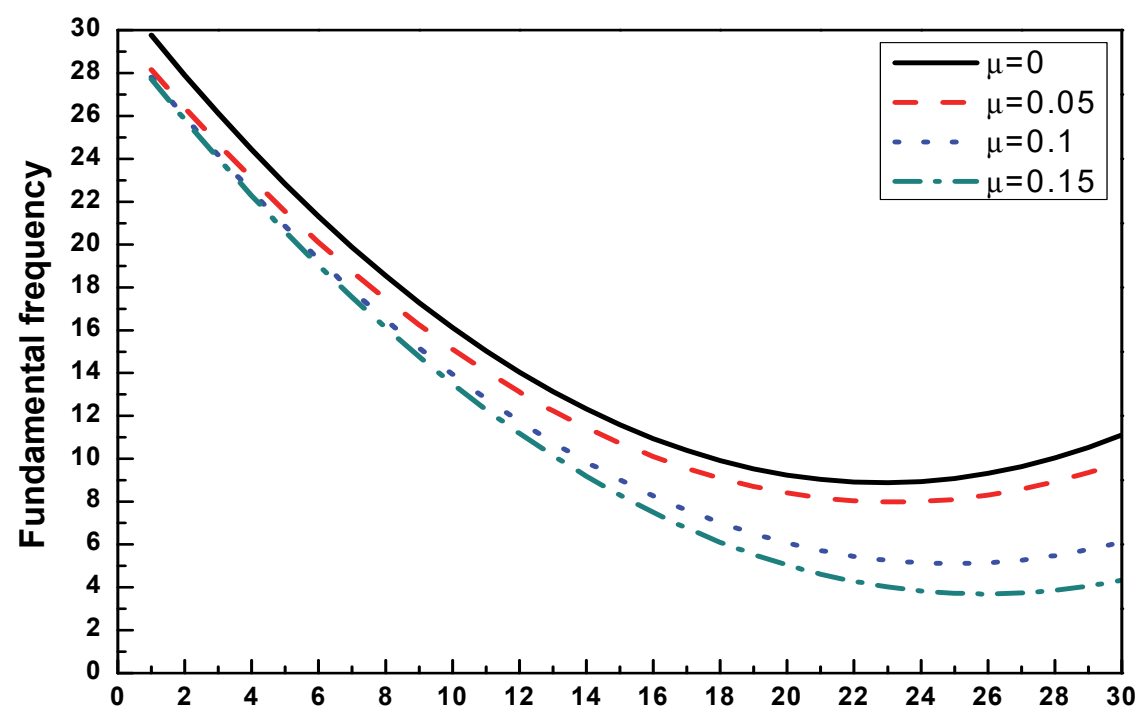

(A) Length-to-thickness ratio
BiTiO3-COFe2O4

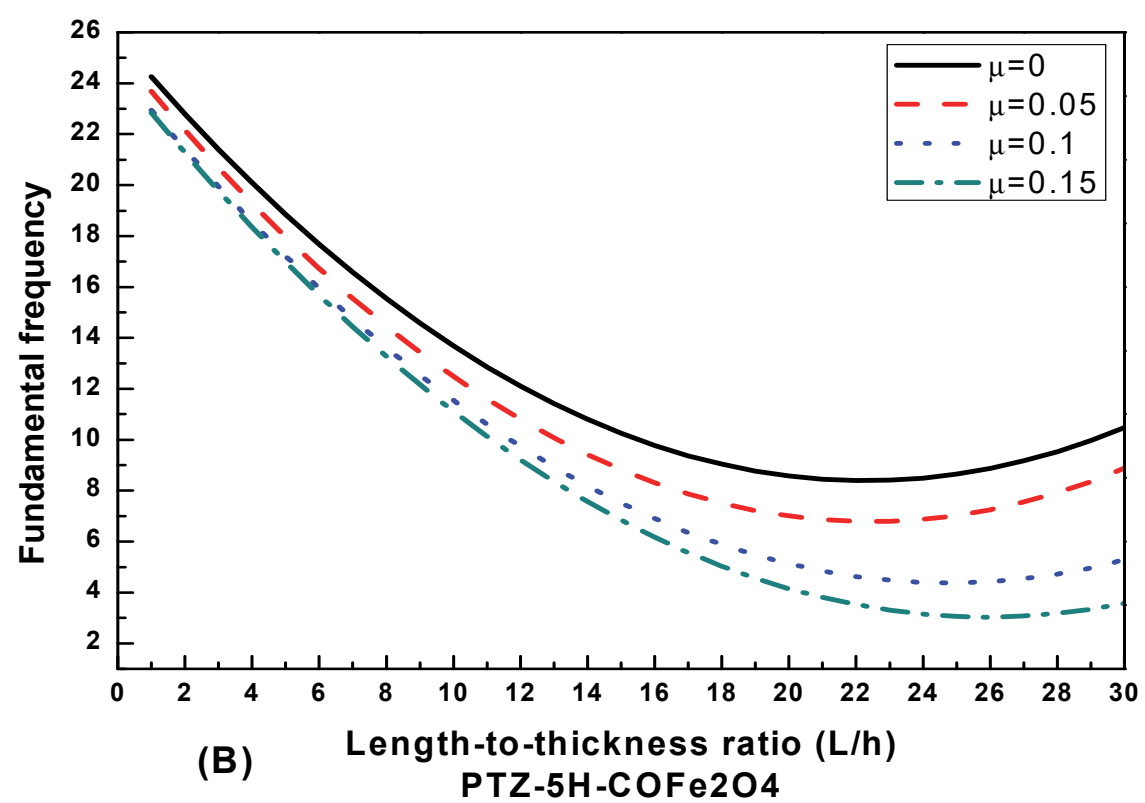

Figure 7. Variation of fundamental frequency with length-to-thickness ratio $(\mathrm{L} / \mathrm{h})$, nonlocal parameter $(\mu)$ and different materials for Hinged-Hinged METE nanobeam.

$$
\left(P_{0}=2, V_{0}=-0.3, \Delta T=100, \mathrm{~A}_{0}=0.02, \mathrm{~h}=2 \mathrm{~nm}, \mathrm{k}_{1}=25, \mathrm{k}_{2}=0.05, \mathrm{k}_{3}=0.15\right)
$$



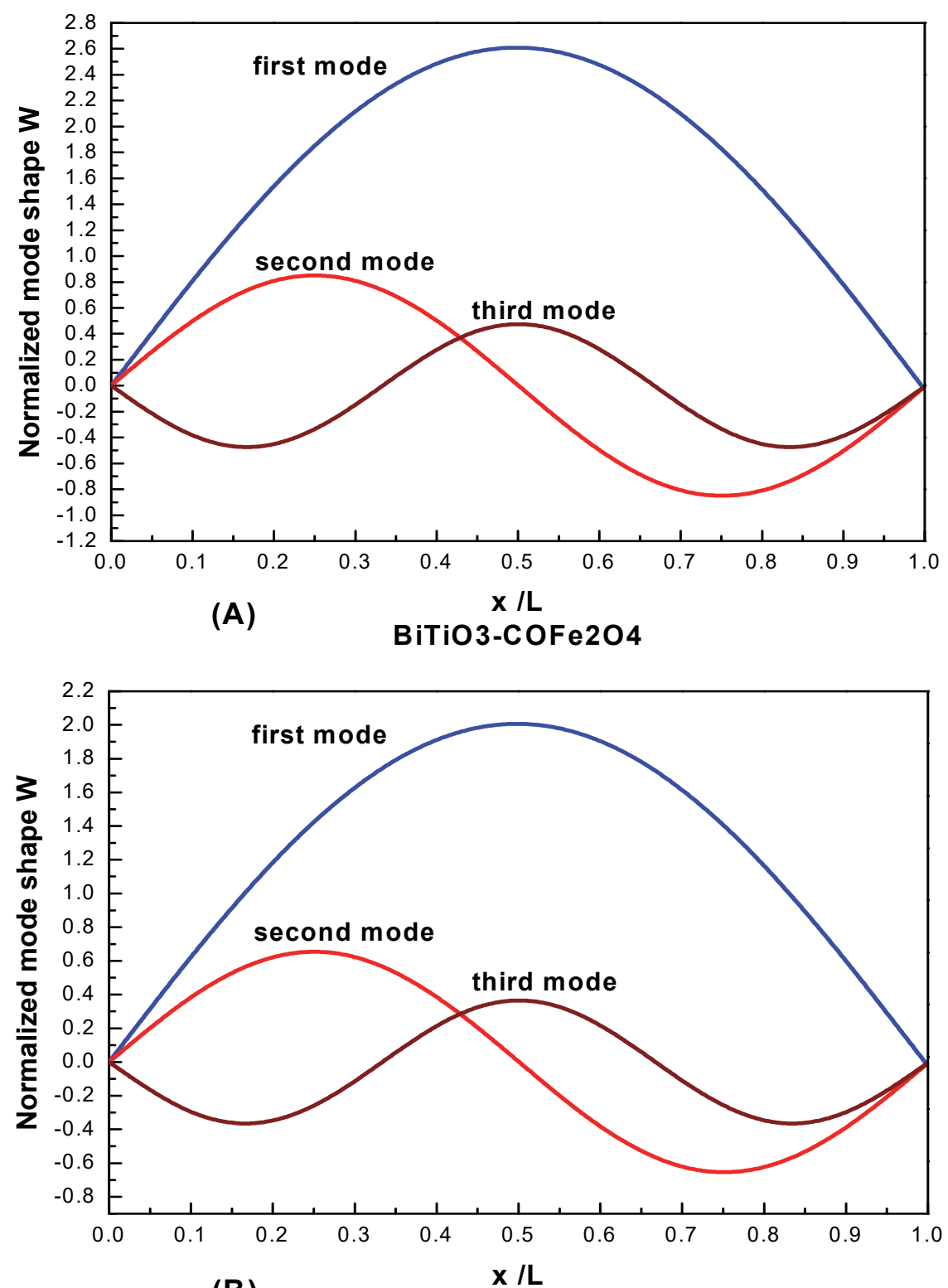

(B)

PTZ-5H-COFe2O4

Figure 8. Variation of normalized mode shape $\mathrm{W}$ with length of nanobeam $\zeta=x / L$ for first three modes at different Material: (A) BiTiO3-COFe2O4; (B) PTZ-5H-COFe2O4 for Hinged-Hinged METE nanobeam.

$$
\left(P_{0}=0, \mathrm{~A}_{0}=0.05, \Delta T=100, \mathrm{~V}_{0}=-0.3, \mathrm{~h}=10 \mathrm{~nm}, \mathrm{~L} / \mathrm{h}=6, \mathrm{k}_{1}=\mathrm{k}_{2}=\mathrm{k}_{3}=0\right)
$$



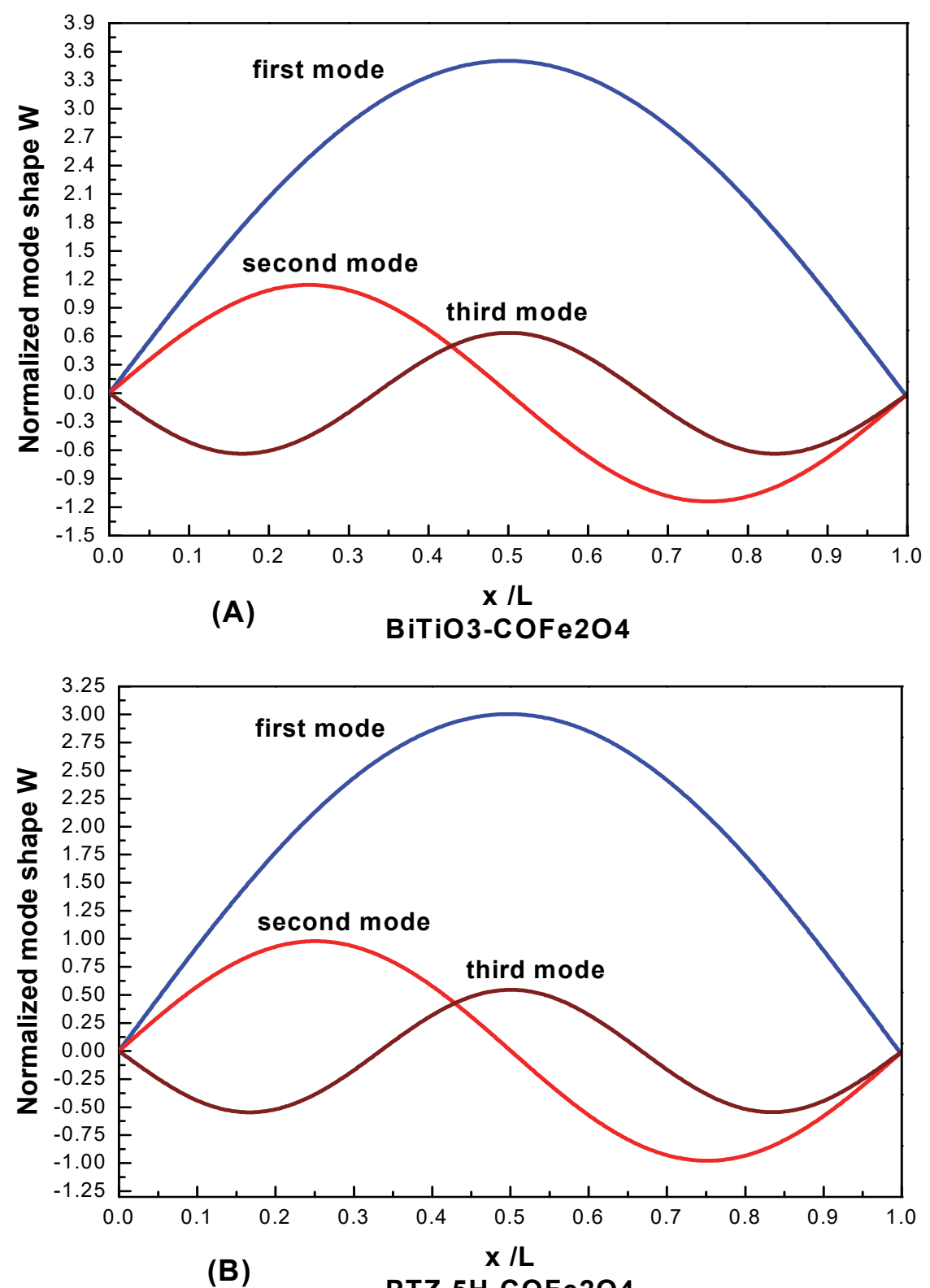

PTZ-5H-COFe2O4

Figure 9. Variation of normalized mode shape $\mathrm{W}$ with length of nanobeam $\zeta=x / L$ for first three modes at different Materials: (A) BiTiO3-COFe2O4; (B) PTZ-5H-COFe2O4 for Hinged-Hinged METE nanobeam.

$$
\left(P_{0}=0, \mathrm{~A}_{0}=0.05, \Delta T=100, \mathrm{~V}_{0}=-0.3, \mathrm{~h}=10 \mathrm{~nm}, \mathrm{~L} / \mathrm{h}=6, \mathrm{k}_{1}=25, \mathrm{k}_{2}=0.05, \mathrm{k}_{3}=0.15\right)
$$




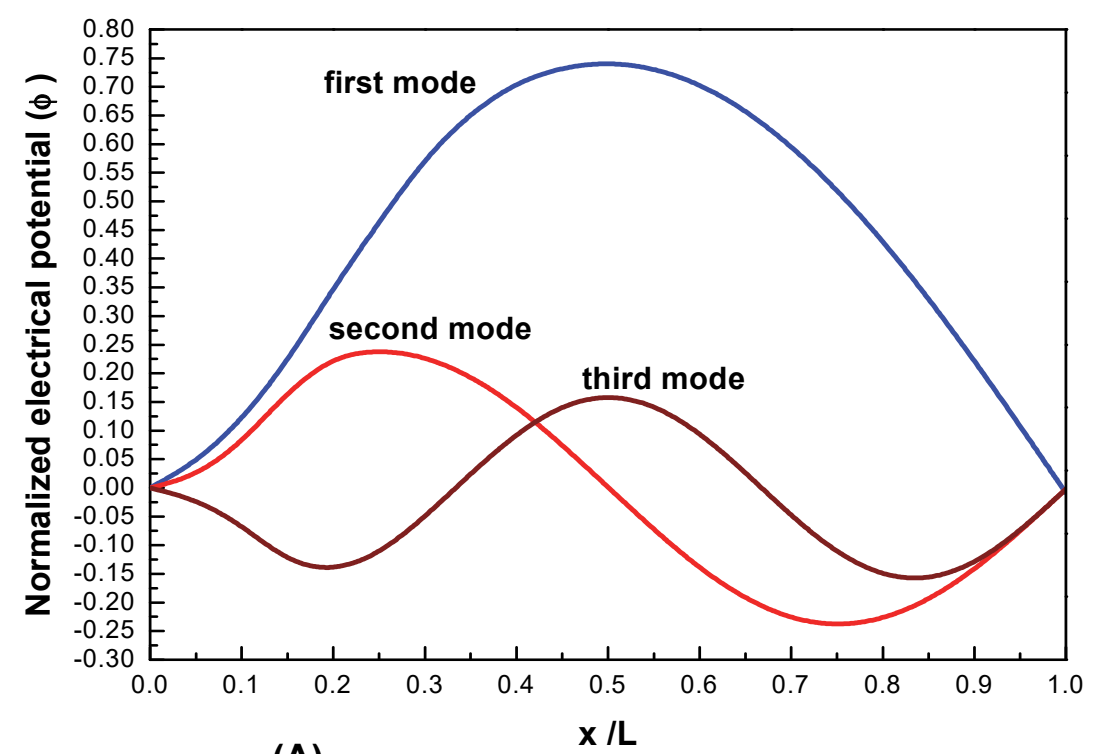

(A)

BiTiO3-COFe2O4

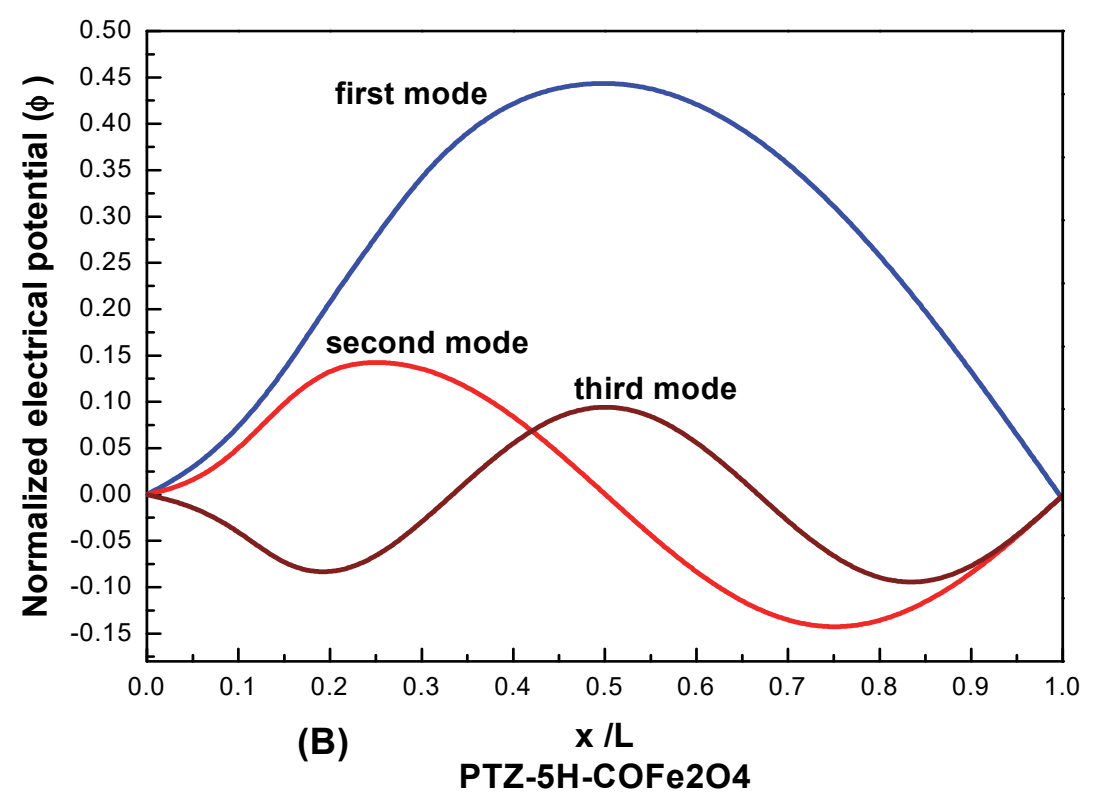

Figure 10. Variation of normalized electrical potential $(\phi)$ with length of nanobeam $\zeta=x / L$ for first three modes at different Materials: (A) BiTiO3-COFe2O4; (B) PTZ-5H-COFe2O4 for Clamped METE nanobeam. $\left(P_{0}=0, \mathrm{~A}_{0}=0.05, \Delta T=100, \mathrm{~V}_{0}=-0.3, \mathrm{~h}=10 \mathrm{~nm}, \mathrm{~L} / \mathrm{h}=6, \mathrm{k}_{1}=\mathrm{k}_{2}=\mathrm{k}_{3}=0\right)$ 

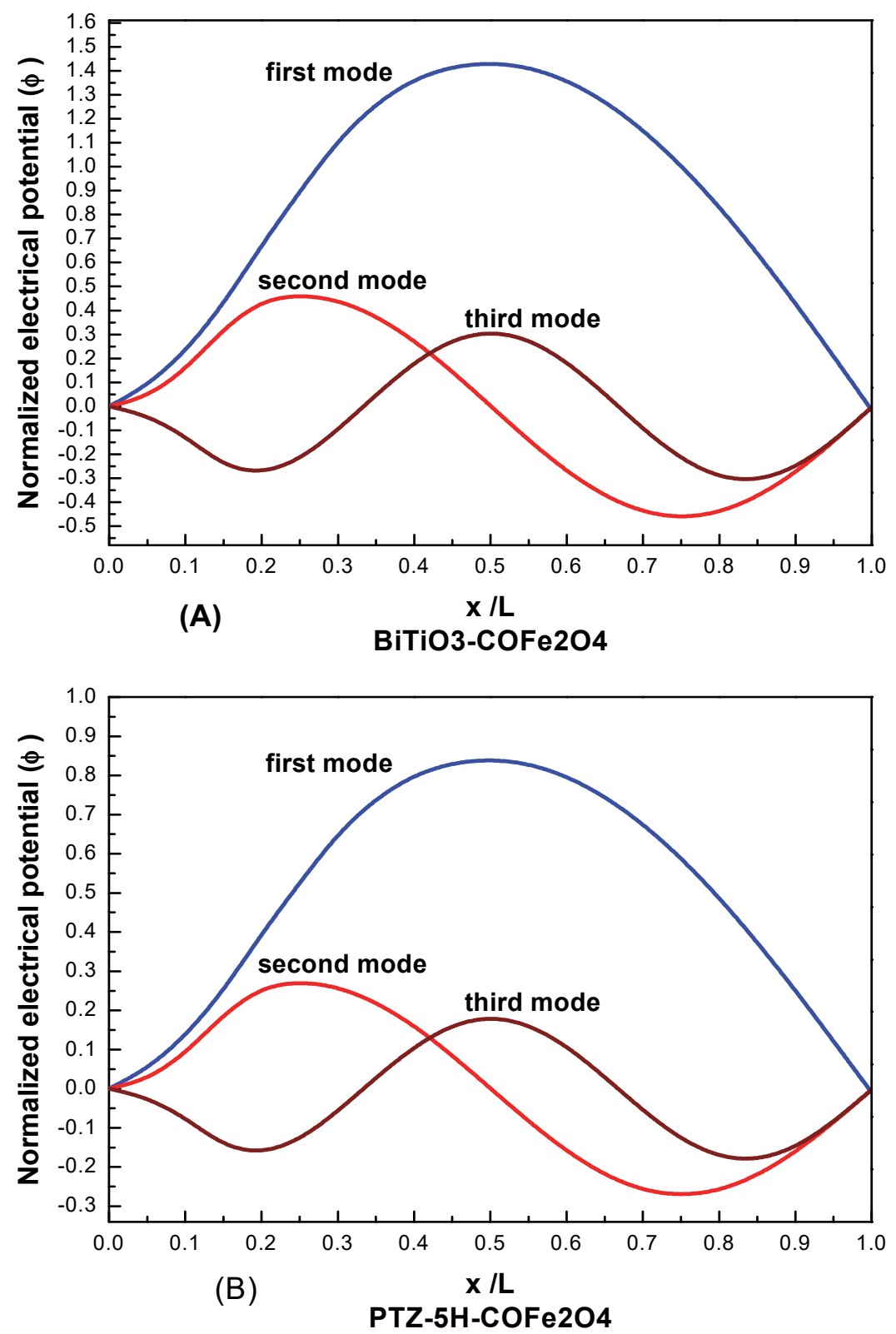

Figure 11. Variation of normalized electrical potential $(\phi)$ with length of nanobeam $\zeta=x / L$ for first three modes at different Materials: (A) BiTiO3-COFe2O4; (B) PTZ-5H-COFe2O4 for Clamped METE nanobeam. $\left(P_{0}=0, \mathrm{~A}_{0}=0.05, \Delta T=100, \mathrm{~V}_{0}=-0.3, \mathrm{~h}=10 \mathrm{~nm}, \mathrm{~L} / \mathrm{h}=6, \mathrm{k}_{1}=25, \mathrm{k}_{2}=0.05, \mathrm{k}_{3}=0.15\right)$

\section{Conclusion}

Three Different Quadrature schemes and Iterative quadrature technique have been successfully applied for vibration analysis of magneto-electro-thermo-elastic nanobeams. MATLAB program is designed for each scheme such that the maximum error (comparing with the previous exact results) is $\leq 10^{-10}$. Also, Execution time for each scheme, is determined. It is concluded that discrete singular convolution differential quadrature method based on regularized Shannon kernel (DSCDQM-RSK) with grid size $\geq 3$, bandwidth $2 \mathrm{M}+1 \geq 3$ and regulization parameter $\sigma=1.75 * \mathrm{~h}_{\mathrm{x}}$ leads to best accurate efficient results to the concerned problem. Based on this scheme, a parametric study is introduced to investigate the influence of different boundary conditions, type of material, linear 
and nonlinear elastic foundation, nonlocal parameter, the length-to-thickness ratio, external electric and magnetic potentials, axial forces, temperature and their effects of the vibrated nanobeam, on results. The main results of our analysis are expressed as:

1. The fundamental frequency increases with increasing linear elastic foundation parameters axial forces and external magnetic potential. Also, the fundamental frequencies depend on the sign and magnitude of the magnetic potential, axial forces and the computations declare that the results do not affect significantly by nonlinear elastic foundation parameter $\mathrm{k} 3$.

2. The fundamental frequency decrease with increasing temperature change, external electric voltage, nonlocal parameter, and length-to-thickness ratio.

3. The amplitudes of displacement $\mathrm{W}$ and electrical potential increase with increasing linear and nonlinear elastic foundation parameters.

4. The fundamental frequency, normalized amplitude and normalized electrical potential for $\mathrm{BiTiO} 3-\mathrm{COFe} 2 \mathrm{O} 4$ material is higher than PTZ-5H-COFe2O4 material.

It is aimed that these results may be useful for the design of smart nanostructures constructed from magnetoelectro-thermo-elastic materials, electromechanical applications and many fields of the industrial revolution. The most important applications of nanobeam is likely to take advantage of their exceptional mechanical, chemical and electrical properties to be used as sensors, resonators and transducers for nanoelectronic and biotechnology applications.

\section{References}

Akgöz, B., \& Civalek, O. (2011). Buckling analysis of cantilever carbon nanotubes using the strain gradient elasticity and modified couple stress theories. Journal of Computational and Theoretical Nanoscience, 8 , 1821-1827. https://doi.org/10.1166/jctn.2011.1888

Ansari, R., Gholami, R., \& Rouhi, H. (2015). Size-dependent nonlinear forced vibration analysis of magnetoelectro-thermo-elastic Timoshenko nanobeams based upon the nonlocal elasticity theory. Compos. Struct., 126, 216-26. https://doi.org/10.1016/j.compstruct.2015.02.068

Arefi, M., \& Zenkour, A. M. (2016). A simplified shear and normal deformations nonlocal theory for bending of functionally graded piezomagnetic sandwich nanobeams in magneto-thermo-electric environment. Journal of Sandwich Structures and Materials, 18, 624-651. https://doi.org/10.1177/1099636216652581

Chang, S. (2000). Differential Quadrature and Its Application in Engineering. Springer-Verlag London Ltd., https://doi.org/10.1007/978-1-4471-0407-0

Chang, T. P. (2013). Deterministic and random vibration analysis of fluid-contacting transversely isotropic magneto-electro-elastic plates. Comput. Fluids, 84, 247-54. https://doi.org/10.1016/j.compfluid.2013.06.009

Civalek, O., \& Kiracioglu, O. (2010). Free vibration analysis of Timoshenko beams by DSC method. Int. J. Numer. Meth. Biomed. Engng., 26, 250-275. https://doi.org/10.1002/cnm.1279

Civalek, O. (2008). Vibration analysis of conical panels using the method of discrete singular convolution. Commun Numer Methods Eng., 24, 169-81. https://doi.org/10.1002/cnm.961

Civalek, O. (2009). Fundamental frequency of isotropic and orthotropic rectangular plates with linearly varying thickness by discrete singular convolution method. Appl Math Model, 33, 3825-3835. https://doi.org/10.1016/j.apm.2008.12.019

Civalek, O. (2017). Free vibration of carbon nanotubes reinforced (CNTR) and functionally graded shells and plates based on FSDT via discrete singular convolution method. Composites Part B, 111, 45-59. https://doi.org/10.1016/j.compositesb.2016.11.030

Fakher, M., \& Shahrokh, H. H. (2017). Bending and free vibration analysis of nanobeams by differential and integral forms of nonlocal strain gradient with Rayleigh-Ritz method. Materials Research Express, 4, 125025. https://doi.org/10.1088/2053-1591/aa9dd6

Foroughi, H., \& Azhari, M. (2014). Mechanical buckling and free vibration of thick functionally graded plates resting on elastic foundation using the higher order B-spline finite strip method. Meccanica, 49, 981-993. https://doi.org/10.1007/s11012-013-9844-2

Huang, D. J., Ding, H. J., \& Chen, W. Q. (2010). Static analysis of anisotropic functionally graded magnetoelectro-elastic beams subjected to arbitrary loading. Eur. J. Mech., 29, 356-69. 
https://doi.org/10.1016/j.euromechsol.2009.12.002

Huang, Y., Luo, Q. Z., \& Li, X. F. (2013). Transverse waves propagating in carbon nanotubes via a higher-order nonlocal beam model. Composite Structures, 95, 328-336. https://doi.org/10.1016/j.compstruct.2012.07.038

Jandaghian, A. A., \& Rahmani, O. (2016a). An analytical Solution for Free Vibration of Piezoelectric nanobeams Based on a Nonlocal Elasticity Theory. Smart Materials and Structures, 32, 143-151. https://doi.org/10.1017/jmech.2015.53

Jandaghian, A. A., \& Rahmani, O. (2016b). Free vibration analysis of magneto-electrothermo elastic nanobeams resting on a Pasternak foundation. Smart Materials and Structures, 25, 035023. https://doi.org/10.1088/0964$1726 / 25 / 3 / 035023$

Jandaghian, A., Jafari, A., \& Rahmani, O. (2013). Exact solution for transient bending of a circular plate integrated with piezoelectric layers. Appl. Math. Modelling, 37, 7154-63. http://doi.org/10.1016/j.apm.2013.02.007: https://doi.org/

Jian, S. P., Yan, L., \& Jie, Y. (2010). A Semi Analytical Method for Nonlinear Vibration of Euler-Bernoulli Beams with General Boundary Conditions. Mathematical Problems in Engineering, 1-17. http://doi.org/10.1155/2010/591786

Ke, L. L., \& Wang, Y. S. (2014). Free vibration of size-dependent magneto-electro-elastic nanobeams based on the nonlocal theory. Physica E, 63, 52-61. https://doi.org/10.1016/j.physe.2014.05.002

Ke, L. L., Wang, Y. S., Yang, J., \& Kitipornchai, S. (2014b). The size dependent vibration of embedded magnetoelectro-elastic cylindrical nanoshells, Smart Mater. Struct., 23, 125036. https://doi.org/10.1088/0964$1726 / 23 / 12 / 125036$

Ke, L., Wang, Y. S., \& Zheng-Dao, W. (2012). Nonlinear vibration of the piezoelectric nanobeams based on the nonlocal theory. Composite Structures, 94, 2038-2047. https://doi.org/10.1016/j.compstruct.2012.01.023

Korkmaz, A., \& İdris, D. (2011). Shock wave simulations using Sinc Differential Quadrature Method. Engineering Computations. International Journal for Computer-Aided Engineering and Software, 28, 654-674. https://doi.org/10.1108/02644401111154619

Li, C. (2014). A nonlocal analytical approach for torsion of cylindrical nanostructures and the existence of higherorder stress and geometric boundaries. Composite Structures, 118, 607-621. https://doi.org/10.1016/j.compstruct.2014.08.008

Li, X. F., \& Wang, B. L. (2009). Vibrational modes of Timoshenko beams at small scales. Applied Physics Letters, 94, 101903. https://doi.org/10.1063/1.3094130

Martin, L. W., Crane, S. P., Chu, Y. H., Holcomb, M. B., Gajek, M., Huijben, M., Yang, C. H., Balke, N., \& Ramesh, R. (2008). Multiferroic and magnetoelectrics: thin films and nanostructures. J. Phys.: Condens.Matter, 20, 434220. https://doi.org/10.1088/0953-8984/20/43/434220

Mercan, K., \& Civalek, O. (2016). DSC method for buckling analysis of boron nitride nanotube (BNNT) surrounded by an elastic matrix. Composite Structures, 143, 300-309. http://doi.org/10.1016/j.compstruct.2016.02.040

Nan, C. W. (1994). Magneto electric effect in composites of piezoelectric and piezomagnetic phases. Phys. Rev. B, 50, 6082-6088. https://doi.org/10.1103/PhysRevB.50.6082

Nan, C.W., Bichurin, M., Dong, S., Viehland, D., \& Srinivasan, G. (2008). Multiferroic magnetoelectric composites: historical perspective, status, and future directions. J. Appl. Phys., 103, 031101, https://doi.org/10.1063/1.2836410

Norouzzadeh, A., \& Ansari, R. (2017). Finite element analysis of nano-scale Timoshenko beams using the integral model of nonlocal elasticity. Phys E., 88, 194-200. https://doi.org/10.1016/j.physe.2017.01.006

Prashanthi, K., Shaibani, P. M., Sohrabi, A., Natarajan, T. S., \& Thundat, T. (2012). Nanoscale magnetoelectric couplinginmultiferroicBiFeO3 nanowires. Phys. Status Solidi $R, 6, \quad 244-246$. https://doi.org/10.1002/pssr.201206135

Ragb, O., Seddek, L. F., \& Matbuly, M. S. (2017). Iterative differential quadrature solutions for Bratu problem. Computers \& Mathematics with Applications, 74(2), 249-257. https://doi.org/10.1016/j.camwa.2017.03.033

Roque, C. M. C., Fidalgo, D. S., Ferreira, A. J. M., \& Reddy, J. N. (2013). A study of a microstructure-dependent composite laminated Timoshenko beam using a modified couple stress theory and a meshless method. 
Composite Structures, 96, 532-537. https://doi.org/10.1016/j.compstruct.2012.09.011

Seçkin, A., \& Sarıgül, A. S. (2009). Free vibration analysis of symmetrically laminated thin composite plates by using discrete singular convolution (DSC) approach: algorithm and verification. J Sound Vib., 315, 197-211. https://doi.org/10.1016/j.jsv.2008.01.061

Shashidhar, S., \& Li. Y. J. (2005). The effective magnetoelectric coefficients of polycrystalline multiferroic composites. Acta Materialia, 53, 4135-4142. https://doi.org/doi:10.1016/j.actamat.2005.05.014

Shen, J. P., \& Li, C. (2017). A semi-continuum-based bending analysis for extreme-thin micro/nano-beams and new proposal for nonlocal differential constitution. Composite Structures, 172, 210-220. https://doi.org/10.1016/j.compstruct.2017.03.070

Shen, Z. B., Li, X. F., Sheng, L. P., \& Tang, G. J. (2012). Transverse vibration of nanotube-based micro-mass sensor via nonlocal Timoshenko beam theory. Computational Materials Science, 53, 340-346. https://doi.org/10.1016/j.commatsci.2011.09.023

Shojaei, M. F., \& Ansari, R. (2017). Variational differential quadrature: A technique to simplify numerical analysis of structures. Applied Mathematical Modelling, 49, 705-738. https://doi.org/10.1016/j.apm.2017.02.052

Şimşek, M., \& Yurtcu, H. (2013). Analytical solutions for bending and buckling of functionally graded nanobeams based on the nonlocal Timoshenko beam theory. Compos.Struct., 97, 378-386. https://doi.org/10.1016/j.compstruct.2012.10.038

Tornabene, F., Dimitri, R., \& Viola, E. (2016). Transient dynamic response of generally shaped arches based on a GDQ-Time-stepping method. Int J Mech Sci., 114, 277-314. https://doi.org/10.1016/j.ijmecsci.2016.05.005

Tornabene, F., Fantuzzi, N., Viola, E., \& Carrera, E. (2014). Static analysis of doubly-curved anisotropic shells and panels using CUF approach, differential geometry and differential quadrature method. Compos Struct., 107, 675-97. https://doi.org/10.1016/j.compstruct.2013.08.038

Wei, G. W. (2001). A new algorithm for solving some mechanical problems. Comput. Methods Appl. Mech. Engrg., 190, 2017-2030. https://doi.org/10.1016/S0045-7825(00)00219-X

Wu, C. P., \& Tsai, Y. H. (2007). Static behavior of functionally graded magneto-electro-elastic shells under electric displacement and magnetic flux. Int. J. Eng. Sci., 45, 744-69, https://doi.org/10.1016/j.ijengsci.2007.05.002

Zhai, J., Xing, Z., Dong, S., Li, J., \& Viehland, D. (2008). Magnetoelectric laminate composites: an overview. J.Am.Ceram.Soc., 91, 351-358. https://doi.org/10.1111/j.1551-2916.2008.02259.x

\section{Copyrights}

Copyright for this article is retained by the author(s), with first publication rights granted to the journal.

This is an open-access article distributed under the terms and conditions of the Creative Commons Attribution license (http://creativecommons.org/licenses/by/4.0/). 